\title{
Dispersion for 1-D Schrödinger and wave equations with BV coefficients
}

\author{
Constantin N. Beli ${ }^{a}$, Liviu I. Ignat ${ }^{\mathrm{a}, \mathrm{b}, *}$, Enrique Zuazua ${ }^{\mathrm{c}, \mathrm{d}}$ \\ a Institute of Mathematics "Simion Stoilow" of the Romanian Academy, 21 Calea Grivitei Street, 010702 Bucharest, Romania \\ ${ }^{\mathrm{b}}$ Faculty of Mathematics and Computer Science, University of Bucharest, 14 Academiei Street, 010014 Bucharest, Romania \\ ${ }^{\text {c } B C A M}$ - Basque Center for Applied Mathematics, Alameda de Mazarredo 14, 48009 Bilbao, Basque Country, Spain \\ d Ikerbasque, Basque Foundation for Science Maria Diaz de Haro 3, 48013, Bilbao, Basque Country, Spain
}

Received 14 May 2014; received in revised form 21 April 2015; accepted 25 June 2015

Available online 26 July 2015

\begin{abstract}
In this paper we analyze the dispersion for one dimensional wave and Schrödinger equations with BV coefficients. In the case of the wave equation we give a complete answer in terms of the variation of the logarithm of the coefficient showing that dispersion occurs if this variation is small enough but it may fail when the variation goes beyond a sharp threshold. For the Schrödinger equation we prove that the dispersion holds under the same smallness assumption on the variation of the coefficient. But, whether dispersion may fail for larger coefficients is unknown for the Schrödinger equation.
\end{abstract}

(c) 2015 L'Association Publications de l'Institut Henri Poincaré. Published by Elsevier B.V. All rights reserved.

Keywords: Schrödinger equation; Wave equation; One space dimension; $B V$ coefficients; Dispersion and Strichartz estimates; Almost periodic functions

\section{Introduction}

In this paper we consider the following two equations with variable coefficients: The one-dimensional wave equation

$$
\begin{cases}v_{t t}(t, x)-\partial_{x}\left(a(x) \partial_{x} v\right)(t, x)=0, & (t, x) \in \mathbb{R}^{2}, \\ v(0, x)=v_{0}(x), v_{t}(0, x)=0, & x \in \mathbb{R},\end{cases}
$$

and the Schrödinger equation

$$
\begin{cases}i u_{t}(t, x)+\partial_{x}\left(a(x) \partial_{x} u\right)(t, x)=0, & (t, x) \in \mathbb{R}^{2}, \\ u(0, x)=u_{0}(x), & x \in \mathbb{R} .\end{cases}
$$

\footnotetext{
* Corresponding author.

E-mail addresses: constantin.beli@imar.ro (C.N. Beli), liviu.ignat@gmail.com (L.I. Ignat), zuazua@bcamath.org (E. Zuazua).

URLs: http://www.imar.ro/ lignat (L.I. Ignat), http://www.bcamath.org/zuazua/ (E. Zuazua).
} 
Along the paper we will consider nonnegative functions $a$ with bounded variation and satisfying the following lower and upper bounds

$$
0<m \leq a(x) \leq M, \quad x \in \mathbb{R} .
$$

The main positive results of this paper are as follows.

Theorem 1.1. For any $a \in B V(\mathbb{R})$ satisfying (1.3) and $\operatorname{Var}(\log (a))<2 \pi$ there exists a positive constant $C(\operatorname{Var}(a)$, $m, M)$ such that the solution $v$ of (1.1) satisfies

$$
\sup _{x \in \mathbb{R}} \int_{\mathbb{R}}|v(t, x)| d t \leq C(\operatorname{Var}(a), m, M)\left\|v_{0}\right\|_{L^{1}(\mathbb{R})} .
$$

Theorem 1.2. For any $a \in B V(\mathbb{R})$ satisfying (1.3) and $\operatorname{Var}(\log (a))<2 \pi$ there exists a positive constant $C(\operatorname{Var}(a)$, $m, M)$ such that the solution $u$ of (1.2) satisfies

$$
\|u(t)\|_{L^{\infty}(\mathbb{R})} \leq \frac{C(\operatorname{Var}(a), m, M)}{\sqrt{t}}\left\|u_{0}\right\|_{L^{1}(\mathbb{R})}
$$

In the case of the wave equation a counterexample can also be established when the total variation of the logarithm of the coefficient is large, showing that our dispersion result above is sharp.

Theorem 1.3. Let be $0<m<M$ and $\alpha \geq 2 \pi$. For any positive number $N$ large enough there exists a piecewise constant function, $m \leq a \leq M$, with $\operatorname{Var}(\log (a))=\alpha$ such that for some $v_{0} \in L^{1}(\mathbb{R})$ the solution $v$ of problem (1.1) satisfies

$$
\sup _{x \in \mathbb{R}} \int_{\mathbb{R}}|v(t, x)| d t \geq N\left\|v_{0}\right\|_{L^{1}(\mathbb{R})} .
$$

Such a counterexample is not available for the Schrödinger equation. Thus, whether the dispersion result in Theorem 1.2 is sharp is an open problem.

Our results are given in terms of the total variation of function $\log (a)$. However under the boundedness assumption above (1.3), $\operatorname{Var}(a)$ and $\operatorname{Var}(\log a)$ are comparable.

The main ideas of the proofs of the above results come from the analysis of wave propagation in multi-layer structures [5, Ch. 3] and [1]. The proof follows mainly the ideas in [1] but with finer resolvent estimates.

We recall that, once the dispersion is established for the solutions of the linear Schrödinger equation, more general space-time estimates can be obtained, namely, the so-called Strichartz estimates

$$
\|u\|_{L^{q}\left(\mathbb{R}, L^{r}(\mathbb{R})\right)} \leq C(q, r)\|\varphi\|_{L^{2}(\mathbb{R})},
$$

for some admissible pairs $(q, r)$. Strichartz estimates for BV coefficients in 1-d without smallness conditions have been established in [2] without making use of the dispersion property. This paper is devoted to investigate under which assumptions the dispersion property still holds.

Estimates similar to these in Theorem 1.1 but integrating on the space variable $x$ instead of time, have been obtained in [4] under a smallness assumption on the $B V$-norm of $\log (a)$. The methods developed in this paper could very likely be useful to further analyze the problems addressed in [4]. But this is still to be done.

The paper is organized as follows. In Section 2 we present some preliminary results from [1] and state two technical lemmas that allow us to improve the results in [1]. In Section 3 we prove the main results stated in the introduction. We point out that the proof of Theorem 1.2 uses previous results from the proof of Theorem 1.1. Section 4 contains the proofs of the two technical lemmas. We will obtain estimates on some almost periodic functions by using some tools from analytical number theory. 


\section{Resolvent estimates on a laminar media}

In this section we collect some previous results from [1], keeping the same notations. Let us consider a partition of the real axis

$$
-\infty=x_{0}<x_{1}<x_{2}<\cdots<x_{n-1}<x_{n}=\infty
$$

and a step function

$$
a(x)=b_{k}^{-2}, \quad x \in I_{k}=\left(x_{k-1}, x_{k}\right), k=1, \ldots, n,
$$

where $1 / M^{2} \leq b_{k} \leq 1 / m^{2}$.

Let us now consider the self-adjoint operator $A=-\partial_{x} a(x) \partial_{x}$ defined from $\left\{h \in H^{1}(\mathbb{R}), a \partial_{x} h \in L^{2}(\mathbb{R})\right\}$ to $L^{2}(\mathbb{R})$. For $\omega \geq 0$ let us consider $R_{\omega}$ its resolvent:

$$
R_{\omega} g=\left(-\partial_{x} a(x) \partial_{x}+\omega^{2} I\right)^{-1} g .
$$

It follows that for $x \in I_{k}=\left(x_{k-1}, x_{k}\right), k=1, \ldots, n$, we have

$$
R_{\omega} g(x)=c_{2 k-1}(\omega) e^{\omega b_{k} x}+c_{2 k}(\omega) e^{-\omega b_{k} x}+b_{k} \int_{I_{k}} \frac{g(y)}{2 \omega} e^{-\omega b_{k}|x-y|} d y,
$$

where $c_{2}=c_{2 n-1}=0$ and the other coefficients are determined by solving the system obtained from the continuity of $R_{\omega} g$ and $a(x) \partial_{x} R_{\omega} g$ at the points $x_{k}, k=1, \ldots, n-1$. It follows that

$$
D_{n}(\omega) C=T
$$

where $C=\left[c_{1}, c_{3}, c_{4}, \ldots, c_{2 n-3}, c_{2 n-2}, c_{2 n}\right]^{\mathrm{T}}, T=\left(t_{1}, \ldots, t_{n-1}\right)^{\mathrm{T}}, t_{k}=\left(t_{k 1}, t_{k 2}\right)^{\mathrm{T}}$,

$$
t_{k}=\left(\begin{array}{l}
-b_{k} \int_{I_{k}} \frac{g(y)}{2 \omega} e^{-\omega b_{k}\left(x_{k}-y\right)} d y+b_{k+1} \int \frac{g(y)}{2 \omega} e^{-\omega b_{k+1}\left(y-x_{k}\right)} d y \\
b_{k} b_{k+1}\left(\int_{I_{k}} \frac{g(y)}{2 \omega} e^{-\omega b_{k}\left(x_{k}-y\right)} d y+\int_{I_{k+1}} \frac{g(y)}{2 \omega} e^{-\omega b_{k+1}\left(y-x_{k}\right)} d y\right)
\end{array}\right), k=1, \ldots, n-1,
$$

and

$$
D_{n}=\left(\begin{array}{cccccccc}
\mathbf{a}_{1} & B_{1} & 0 & 0 & 0 & 0 & 0 & 0 \\
0 & A_{2} & B_{2} & 0 & 0 & 0 & 0 & 0 \\
0 & 0 & A_{3} & B_{3} & 0 & 0 & 0 & 0 \\
- & - & - & - & - & - & - & - \\
0 & 0 & 0 & 0 & A_{n-3} & B_{n-3} & 0 & 0 \\
0 & 0 & 0 & 0 & 0 & A_{n-2} & B_{n-2} & 0 \\
0 & 0 & 0 & 0 & 0 & 0 & A_{n-1} & \mathbf{b}_{n-1}
\end{array}\right)
$$

with

$$
\begin{aligned}
& \mathbf{a}_{1}=\left(\begin{array}{c}
e^{\omega b_{1} x_{1}} \\
b_{2} e^{\omega b_{1} x_{1}}
\end{array}\right), \quad \mathbf{b}_{n-1}=\left(\begin{array}{c}
-e^{-\omega b_{n} x_{n-1}} \\
b_{n-1} e^{-\omega b_{n} x_{n-1}}
\end{array}\right), \\
& A_{k}=\left(\begin{array}{cc}
e^{\omega b_{k} x_{k}} & e^{-\omega b_{k} x_{k}} \\
b_{k+1} e^{\omega b_{k} x_{k}} & -b_{k+1} e^{-\omega b_{k} x_{k}}
\end{array}\right), B_{k}=\left(\begin{array}{cc}
-e^{\omega b_{k+1} x_{k}} & -e^{-\omega b_{k+1} x_{k}} \\
-b_{k} e^{\omega b_{k+1} x_{k}} & b_{k} e^{-\omega b_{k+1} x_{k}}
\end{array}\right) .
\end{aligned}
$$

For technical reasons we introduce the matrix $\tilde{D}_{n}$ which has the same structure as $D_{n}$ but replacing vector $\mathbf{b}_{n-1}$ with

$$
\tilde{\mathbf{b}}_{n-1}=\left(\begin{array}{c}
-e^{\omega b_{n} x_{n-1}} \\
-b_{n-1} e^{\omega b_{n} x_{n-1}}
\end{array}\right) \text {. }
$$


We point out that the vectors $\mathbf{b}_{n-1}, \tilde{\mathbf{b}}_{n-1}$ appearing in $D_{n}$ and $\tilde{D}_{n}$ are given by the second and respectively first column of $B_{n-1}$.

Let us introduce now the reflection coefficients

$$
d_{k-1}=\frac{b_{k-1}-b_{k}}{b_{k-1}+b_{k}}, k=2, \ldots, n
$$

and the functions $Q_{k}, k=1, \ldots, n$, defined as follows: $Q_{1}(\omega) \equiv 0$ and

$$
Q_{k}(\omega)= \begin{cases}e^{-2 \omega b_{k}\left(x_{k}-x_{k-1}\right)} \frac{-d_{k-1}+Q_{k-1}(\omega)}{1-d_{k-1} Q_{k-1}(\omega)}, & k=2, \ldots, n-1, \\ e^{2 \omega b_{k} x_{k-1}} \frac{-d_{k-1}+Q_{k-1}(\omega)}{1-d_{k-1} Q_{k-1}(\omega)}, & k=n .\end{cases}
$$

It follows that for $2 \leq k \leq n$

$$
Q_{k}(\omega)= \begin{cases}e^{-2 \omega b_{k} x_{k}} \frac{\operatorname{det} \tilde{D}_{k}(\omega)}{\operatorname{det} D_{k}(\omega)}, & k=2, \ldots, n-1, \\ \frac{\operatorname{det} \tilde{D}_{k}(\omega)}{\operatorname{det} D_{k}(\omega)}, & k=n,\end{cases}
$$

and for any $2 \leq k \leq n$

$$
\operatorname{det} D_{k}(\omega)=\prod_{j=1}^{k-1}\left(b_{j}+b_{j+1}\right) e^{\omega\left(b_{j}-b_{j+1}\right) x_{j}}\left(1-d_{j} Q_{j}(\omega)\right) .
$$

It has been proved in [1] that there exists a $\delta>0$ such that for any $\omega \in \mathbb{C}$ with $\Re(\omega)>-\delta$ we have $\left|Q_{k}(\omega)\right|<1, k=$ $2, \ldots, n$. It implies that $\left(\operatorname{det} D_{n}(\omega)\right)^{-1}$ is uniformly bounded in the same region of the complex plane and moreover $\omega R_{\omega} u_{0}$ can be analytically continued. In the case when the coefficient $a$ is as in (2.8) the spectral calculus gives us the following representation of the solutions of equations (1.1) and (1.2).

Lemma 2.1. The solution of the wave equation (1.1) verifies

$$
v(t, x) \mathbf{1}_{\{t>0\}}=\frac{i}{2 \pi} \int_{-\infty}^{\infty} e^{i t \omega} \omega\left(R_{i \omega} v_{0}\right)(x) d \omega .
$$

Lemma 2.2. The solution of the Schrödinger equation (1.2) verifies

$$
u(t, x)=\frac{1}{i \pi} \int_{-\infty}^{\infty} e^{-i t \omega^{2}} \omega\left(R_{i \omega} u_{0}\right)(x) d \omega .
$$

For completeness we prove these lemmas.

Proof of Lemma 2.1. Set $v_{1}(t)=v(t) \mathbf{1}_{\{t>0\}}$. It follows that $v_{1}$ satisfies

$$
\partial_{t t} v_{1}-\partial_{x}\left(a(x) \partial_{x} v_{1}\right)=\partial_{t} \delta_{0} f .
$$

Since $v_{1}(t)$ is supported on $(0, \infty)$ it follows that the Fourier transform in time variable of $v_{1}$ is holomorphic in the

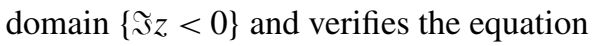

$$
\left(-z^{2}-\partial_{x}\left(a(x) \partial_{x}\right) \hat{v}_{1}(z, \cdot)=i z f(\cdot), \quad \Im(z)<0 .\right.
$$

Taking $z=\omega-i \varepsilon, \omega \in \mathbb{R}, \varepsilon>0$ small enough we obtain that

$$
\hat{v}_{1}(\omega-i \varepsilon)=i(\omega-i \varepsilon) R_{i(\omega-i \varepsilon)} f .
$$


Using the inverse Fourier transform we get

$$
v_{1}(t)=\frac{1}{2 \pi} \int_{\mathbb{R}} e^{i t \omega} i(\omega-i \varepsilon) R_{i(\omega-i \varepsilon)} f d \omega .
$$

Since $\omega R_{\omega}$ can be analytically continued on $\{\Re z>-\delta\}$ we obtain the desired result.

A similar argument shows that

$$
v(t) \mathbf{1}_{\{t<0\}}=-\frac{1}{2 \pi} \int_{\mathbb{R}} e^{i t \omega} i(\omega+i \varepsilon) R_{i(\omega+i \varepsilon)} f d \omega .
$$

The proof is now complete.

Proof of Lemma 2.2. Using the identity

$$
\begin{aligned}
h(\lambda) & =\lim _{\varepsilon \downarrow 0} \frac{1}{2 \pi i} \int_{\mathbb{R}} h(s)\left(\frac{1}{\lambda-(s-i \varepsilon)}-\frac{1}{\lambda-(s+i \varepsilon)}\right) d s \\
& =\frac{1}{2 \pi i} \int_{\mathbb{R}} h(s)\left(\frac{1}{\lambda-(s-i 0)}-\frac{1}{\lambda-(s+i 0)}\right) d s,
\end{aligned}
$$

classical spectral calculus gives us that

$$
u(t, x)=e^{-i t A}=\frac{1}{2 \pi i} \int_{\mathbb{R}} e^{-i t s}\left((A-(s-i 0))^{-1}-(A-(s+i 0))^{-1}\right) u_{0} d s .
$$

Since $\sigma(A)=(0, \infty)$ we have that $(A+z)^{-1}$ is analytic on $\mathbb{C} \backslash(-\infty, 0)$ and that

$$
\left(A+\left(-\tau^{2}+i 0\right)\right)^{-1}=R_{i|\tau|+0}, \quad\left(A+\left(-\tau^{2}-i 0\right)\right)^{-1}=R_{-i|\tau|+0} .
$$

Then

$$
\begin{aligned}
u(t, x) & =\frac{1}{2 \pi i} \int_{0}^{\infty} e^{-i t s}\left((A-(s-i 0))^{-1}-(A-(s+i 0))^{-1}\right) u_{0} d s \\
& =\frac{1}{\pi i} \int_{0}^{\infty} e^{-i t \tau^{2}} \tau\left(\left(A-\left(\tau^{2}-i 0\right)\right)^{-1}-\left(A-\left(\tau^{2}+i 0\right)\right)^{-1}\right) u_{0} d \tau \\
& =\frac{1}{\pi i} \int_{0}^{\infty} e^{-i t \tau^{2}} \tau\left(R_{i \tau+0} u_{0}-R_{-i \tau+0} u_{0}\right) d \tau \\
& =\frac{1}{\pi i} \int_{-\infty}^{\infty} e^{-i t \tau^{2}} \tau R_{i \tau+0} u_{0} d \tau .
\end{aligned}
$$

Using now that $\omega R_{\omega}$ can be analytically continued on $\{\Re z>-\delta\}$ we obtain the desired result.

In the following we denote by $\hat{f}$ and $f^{\vee}$ the Fourier and the inverse Fourier transform of the function $f$ :

$$
\hat{f}(\xi)=\int_{\mathbb{R}} f(x) e^{-i x \xi} d x, \quad f^{\vee}(x)=\frac{1}{2 \pi} \int_{\mathbb{R}} f(\xi) e^{i \xi x} d \xi .
$$

The proof of the main results of this paper requires the theory of almost periodic functions. A function $f: \mathbb{R} \rightarrow \mathbb{C}$ is said to be almost-periodic if it can be represented as 


$$
f(t)=\sum_{n} c_{n} e^{i \lambda_{n} t},
$$

and the following norm satisfies

$$
\|f\|_{A P}=\sum_{n}\left|c_{n}\right|<\infty
$$

It is easy to see that the space of almost periodic functions is an algebra. For more details on the properties of these functions we refer to [3].

As observed in [1], the function $\operatorname{det} D_{n}(i \omega)$ is an almost periodic function. The same property is satisfied by $1 / \operatorname{det} D_{n}(i \omega)$ even if this property is not trivial (see [1, Section 2.2]).

Here, in addition to the results in [1], we will compute exactly the coefficients $c_{k}$ in terms of vector $T$ and sequence $\left\{Q_{k}\right\}_{k=1}^{n}$ by solving the system $D_{n}(\omega) C=T$ (see Section 3 below). The argument in [1] only uses the fact that, since $C$ is a solution of the above system, then its components are finite sums of the terms in the vector $T$. Also, instead of using the results in [1, Section 2.2] we control in a finer way the sequence $\left\{Q_{k}\right\}_{k=1}^{n}$ introduced in (2.11) and prove the following two key lemmas.

Lemma 2.3. Let us consider two sequences of real numbers $\left(c_{n}\right)_{n \geq 1}$ and $\left(d_{n}\right)_{n \geq 1}$ with $\left|d_{n}\right| \leq d<1$ satisfying

$$
\sum_{n \geq 1} \operatorname{arctanh}\left|d_{n}\right|<\frac{\pi}{2}
$$

We also consider the following sequence of functions $Q_{1}(i \omega) \equiv 0$ and

$$
Q_{k}(i \omega)=e^{i c_{k-1} \omega} \frac{-d_{k-1}+Q_{k-1}(i \omega)}{1-d_{k-1} Q_{k-1}(i \omega)}, \omega \in \mathbb{R}, k \geq 2 .
$$

Then for any $n \geq 2$ the following holds

$$
\left\|Q_{n}\right\|_{A P} \leq \tan \left(\sum_{k \geq 1} \operatorname{arctanh}\left|d_{k}\right|\right) .
$$

Moreover, Lemma 2.3 is the best result possible in the following sense:

Lemma 2.4. Let $\alpha>0$ and $0<d<1$. For any $\varepsilon>0$ and any $N>0$ there exist a positive integer $n$ and a sequence $0<\tilde{d}_{k} \leq d, k=1, \ldots, n-1$, with

$$
\sum_{k=1}^{n-1} \operatorname{arctanh} \tilde{d}_{k}=\alpha
$$

such that for any sequence $\left\{c_{k}\right\}_{k=1}^{n-1}$ of numbers linearly independent over the rationals and any sequence $\left\{d_{k}\right\}_{k=1}^{n-1}$ with $\left|d_{k}\right|=\tilde{d}_{k}$ the sequence $\left\{Q_{k}\right\}_{k=1}^{n}$ defined in (2.16) satisfies

$$
\begin{aligned}
& \left\|Q_{n}\right\|_{A P} \geq \tan \alpha-\varepsilon \quad \text { if } \alpha<\pi / 2 \quad \text { and } \\
& \left\|Q_{n}\right\|_{A P} \geq N \quad \text { if } \alpha \geq \pi / 2 .
\end{aligned}
$$

Remark 2.1. Let us remark that the reflection coefficients $d_{k}$ in (2.10) can be rewritten as

$$
d_{k}=\tanh \left(\frac{\log \left(b_{k-1}\right)-\log \left(b_{k}\right)}{2}\right) \text {. }
$$

Since $|\tanh x|=\tanh |x|$ we have

$$
\left|d_{k}\right|=\tanh \left|\frac{\log \left(b_{k-1}\right)-\log \left(b_{k}\right)}{2}\right| .
$$


As a consequence:

$$
\sum_{k \geq 1} \operatorname{arctanh}\left|d_{k}\right|=\sum_{k \geq 1}\left|\frac{\log \left(b_{k-1}\right)-\log \left(b_{k}\right)}{2}\right|=\frac{\operatorname{Var}(\log (a))}{4} .
$$

Also, since $a$ satisfies (1.3) we also have

$$
\frac{\operatorname{Var}(a)}{M} \leq \operatorname{Var}(\log a) \leq \frac{\operatorname{Var}(a)}{m} .
$$

With Lemmas 2.3 and 2.4 we can prove Theorems 1.1 and 1.3. Theorem 1.2 will be a consequence of Theorem 1.1.

Let us now comment on how these lemmas apply to obtain the main results in this paper. The key point in the proof of Theorem 1.1 is, as we will see in Section 3, that for a step function $a$ as in (2.7) and (2.8) the following holds

$$
\sup _{v_{0} \in L^{1}(\mathbb{R})} \frac{\sup _{x \in \mathbb{R}} \int_{\mathbb{R}}|v(t, x)| d t}{\left\|v_{0}\right\|_{L^{1}(\mathbb{R})}} \simeq \sup _{v_{0} \in L^{1}(\mathbb{R})} \frac{\left.\|\left(Q_{n}(i \omega) \widehat{v}_{0}\right)^{\vee}\right) \|_{L^{1}(\mathbb{R})}}{\left\|v_{0}\right\|_{L^{1}(\mathbb{R})}}=\left\|Q_{n}\right\|_{A P .}
$$

Thus the results given by Lemmas 2.3 and 2.4 on the $A P$-norm of $Q_{n}$ provide results for the behavior of the solutions of the wave equation (1.1).

In the case of the Schrödinger equation (1.2), using the same arguments as in the case of the wave equation, we have that

$$
\sup _{u_{0} \in L^{1}(\mathbb{R})} \frac{t^{1 / 2}\|u(t)\|_{L^{\infty}(\mathbb{R})}}{\left\|u_{0}\right\|_{L^{1}(\mathbb{R})}} \simeq \sup _{u_{0} \in L^{1}(\mathbb{R})} \frac{t^{1 / 2}\left\|\left(e^{i t \omega^{2}} Q_{n}(i \omega) \widehat{u}_{0}\right)^{\vee}\right\|_{L^{\infty}(\mathbb{R})}}{\left\|u_{0}\right\|_{L^{1}(\mathbb{R})}} .
$$

Applying Young's inequality it is immediate that for all $t>0$ the following holds

$$
\sup _{u_{0} \in L^{1}(\mathbb{R})} \frac{t^{1 / 2}\left\|\left(e^{i t \omega^{2}} Q_{n}(i \omega) \widehat{u}_{0}\right)^{\vee}\right\|_{L^{\infty}(\mathbb{R})}}{\left\|u_{0}\right\|_{L^{1}(\mathbb{R})}} \leq \sup _{v_{0} \in L^{1}(\mathbb{R})} \frac{\left\|\left(Q_{n}(i \omega) \widehat{v}_{0}\right)^{\vee}\right\|_{L^{1}(\mathbb{R})}}{\left\|v_{0}\right\|_{L^{1}(\mathbb{R})}} \leq\left\|Q_{n}\right\|_{A P .} .
$$

However, we cannot say that the right hand side in (2.24) is comparable with $\left\|Q_{n}\right\|_{A P}$. This is why we have only a positive result when the $B V$-norm of the coefficient $a$ is small. The optimality of the result in Theorem 1.2 is still an open problem.

\section{Proof of the main results}

The aim of this section is to prove the main results of this paper. We first concentrate on the case of the wave equation (1.1). We will prove that the solution of equation (1.1) satisfies

$$
\sup _{x \in \mathbb{R}} \int_{\mathbb{R}}|v(t, x)| d t \leq C(\operatorname{Var}(a), m, M)\left\|v_{0}\right\|_{L^{1}(\mathbb{R})} .
$$

An argument similar to the one in [2, Prop. 1.3] shows that it is sufficient to prove results for piecewise constant functions $a$ taking a finite number of values. Let us consider a laminar medium as in Section 2. The key point in our proof is that the above estimate is equivalent with the fact that $Q_{n}(i \omega)$ is an $L^{1}(\mathbb{R})$-Fourier multiplier and the norm of $Q_{n}$ as an $L^{1}(\mathbb{R})$-multiplier can be estimated in terms of the variation of $\log (a)$.

Proof of Theorem 1.1. Using the spectral formula (2.13) and the representation of the resolvent $R_{i \omega}$ obtained in the previous section, for any $x \in I_{k}$ and $t>0$, the solution $v$ of equation (1.1) can be written as

$$
\begin{aligned}
v(t, x) & =\int_{\mathbb{R}}\left(i \omega c_{2 k-1}(i \omega) e^{i \omega\left(t+b_{k} x\right)}+i \omega c_{2 k}(i \omega) e^{i \omega\left(t-b_{k} x\right)}\right) \frac{d \omega}{2 \pi}+\frac{b_{k}}{4 \pi} \int_{\mathbb{R}} e^{i t \omega} \int_{I_{k}} v_{0}(y) e^{-i \omega b_{k}|x-y|} d y d \omega \\
& =\left(i \omega c_{2 k-1}(i \omega)\right)^{\vee}\left(t+b_{k} x\right)+\left(i \omega c_{2 k}(i \omega)\right)^{\vee}\left(t-b_{k} x\right)+\tilde{v}(t, x)
\end{aligned}
$$


where

$$
\begin{aligned}
\tilde{v}(t, x)= & \frac{b_{k}}{4 \pi} \int_{\mathbb{R}} e^{i t \omega} \int_{I_{k}}\left(v_{0} \mathbf{1}_{\left.\left\{x_{k-1}<y<x\right)\right\}}\right)(y) e^{-i \omega b_{k}(x-y)} d y d \omega \\
& +\frac{b_{k}}{4 \pi} \int_{\mathbb{R}} e^{i t \omega} \int_{I_{k}}\left(v_{0} \mathbf{1}_{\left.\left\{x<y<x_{k}\right)\right\}}\right)(y) e^{-i \omega b_{k}(y-x)} d y d \omega \\
= & \frac{1}{2} \int_{\mathbb{R}} e^{i \omega\left(t / b_{k}-x\right)}\left(v_{0} \mathbf{1}_{\left.\left\{x_{k-1}<y<x\right)\right\}}\right)^{\vee}(\omega) d \omega+\frac{1}{2} \int_{\mathbb{R}} e^{i \omega\left(t / b_{k}+x\right)}\left(v_{0} \mathbf{1}_{\left.\left\{x<y<x_{k}\right)\right\}}\right)^{\wedge}(\omega) d \omega \\
= & \frac{1}{2}\left(v_{0} \mathbf{1}_{\left.\left\{x_{k-1}<y<x\right)\right\}}\right)\left(x-\frac{t}{b_{k}}\right)+\frac{1}{2}\left(v_{0} \mathbf{1}_{\left.\left\{x<y<x_{k}\right)\right\}}\right)\left(x+\frac{t}{b_{k}}\right) .
\end{aligned}
$$

It is easy to see that

$$
\int_{\mathbb{R}}|\tilde{v}(t, x)| d t \leq b_{k}\left\|v_{0}\right\|_{L^{1}(\mathbb{R})} \leq m^{-2}\left\|v_{0}\right\|_{L^{1}(\mathbb{R})} .
$$

Since for $t<0, v(t, x)=v(-t, x)$ we have

$$
\int_{\mathbb{R}}|v(t, x)| d t=2 \int_{0}^{\infty}|v(t, x)| d t .
$$

Hence, in order to prove estimate (3.1), it remains to show that for any $j=1, \ldots, n$ and for any $x \in I_{j}$ the following holds:

$$
\int_{\mathbb{R}}\left|\left(i \omega c_{2 j-1}(i \omega)\right)^{\vee}\left(t+b_{j} x\right)+\left(i \omega c_{2 j}(i \omega)\right)^{\vee}\left(t-b_{j} x\right)\right| d t \leq C(\operatorname{Var}(a), m, M)\left\|v_{0}\right\|_{L^{1}(\mathbb{R})} .
$$

In fact we will prove a stronger estimate: for any $1 \leq j \leq n-1$ the following holds:

$$
\int_{\mathbb{R}}\left(\left|\left(i \omega c_{2 j-1}(i \omega)\right)^{\vee}(x)\right|+\left|\left(i \omega c_{2 j}(i \omega)\right)^{\vee}(x)\right|\right) d x \leq C(\operatorname{Var}(a), m, M)\left\|v_{0}\right\|_{L^{1}(\mathbb{R})} .
$$

We also remark that since $c_{2}=c_{2 n-1}=0$, when $j \in\{1, n-1\}$, the two estimates (3.3) and (3.4) are the same. Estimate (3.4) is the key not only in the proof of Theorem 1.1 but also in the one of Theorem 1.2. Moreover, we point out that it is sufficient to consider $v_{0}$ to be supported in one of the intervals $I_{k}, k=1, \ldots, n$ since by linearity the result extends to any function $v_{0} \in L^{1}(\mathbb{R})$.

Since there is a strong connection between the c's and $t$ 's we observe that for $k=1, \ldots, n-1$,

$$
i \omega t_{k, 1}(i \omega)=-\frac{b_{k}}{2} e^{-i \omega b_{k} x_{k}} \widehat{v_{0} \mathbf{1}_{I_{k}}}\left(-b_{k} \omega\right)+\frac{b_{k+1}}{2} e^{-i \omega b_{k+1} x_{k}} \widehat{v_{0} \mathbf{1}_{I_{k+1}}}\left(b_{k+1} \omega\right)
$$

and

$$
i \omega t_{k, 2}(i \omega)=\frac{b_{k} b_{k+1}}{2}\left(e^{-i \omega b_{k} x_{k}} \widehat{v_{0} \mathbf{1}_{I_{k}}}\left(-b_{k} \omega\right)+e^{-i \omega b_{k+1} x_{k}} \widehat{v_{0} \mathbf{1}_{I_{k+1}}}\left(b_{k+1} \omega\right)\right) .
$$

An immediate consequence is that

$$
\int_{\mathbb{R}}\left|\left(i \omega t_{k, 1}(i \omega)\right)^{\vee}(x)\right| d x \leq \frac{\left|b_{k}\right|+\left|b_{k+1}\right|}{2} \int_{\mathbb{R}}\left|v_{0}\right| \leq c(m) \int_{\mathbb{R}}\left|v_{0}\right|
$$

and

$$
\int_{\mathbb{R}}\left|\left(i \omega t_{k, 2}(i \omega)\right)^{\vee}(x)\right| d x \leq\left|b_{k}\right|\left|b_{k+1}\right| \int_{\mathbb{R}}\left|v_{0}\right| \leq c(m) \int_{\mathbb{R}}\left|v_{0}\right| .
$$


The main steps in the proof of (3.4) are the following:

- Prove (3.4) for $j=n$ and $\operatorname{supp} v_{0} \subset I_{n}$.

- Prove (3.4) for $j=n$ and supp $v_{0} \subset I_{1}$.

By symmetry the same holds for $j \in\{1, n\}$ and supp $v_{0} \subset I_{1} \cup I_{n}$.

- Prove (3.4) for $j=n$ and $\operatorname{supp} v_{0} \subset I_{k}, 2 \leq k \leq n-1$.

- Prove (3.4) for $j \in\{k, \ldots, n-1\}$ and $\operatorname{supp} v_{0} \subset I_{k}$ with $2 \leq k \leq n-1$.

By symmetry the same holds for $x \in I_{j}$ and supp $v_{0} \subset I_{k}, 2 \leq k \leq n-1$ with $1 \leq j \leq k-1$.

Case 1. Computing $c_{2 n}$ when $\operatorname{supp} v_{0} \subset I_{n}$. In this case all $t_{k}, k=1, \ldots, n-2$, vanish. Moreover $t_{n-1,2}=$ $b_{n-1} t_{n-1,1}$. It follows that

$$
c_{2 n}(i \omega)=\left(\operatorname{det} D_{n}(i \omega)\right)^{-1}\left|\begin{array}{cccccccc}
\mathbf{a}_{1} & B_{1} & 0 & 0 & 0 & 0 & 0 & 0 \\
0 & A_{2} & B_{2} & 0 & 0 & 0 & 0 & 0 \\
0 & 0 & A_{3} & B_{3} & 0 & 0 & 0 & 0 \\
- & - & - & - & - & - & - & - \\
0 & 0 & 0 & 0 & A_{n-3} & B_{n-3} & 0 & 0 \\
0 & 0 & 0 & 0 & 0 & A_{n-2} & B_{n-2} & 0 \\
0 & 0 & 0 & 0 & 0 & 0 & A_{n-1} & t_{n-1}
\end{array}\right| .
$$

Developing over the last two lines the above determinant we obtain

$$
\begin{aligned}
c_{2 n}(i \omega)= & -\left|\begin{array}{cc}
e^{i \omega b_{n-1} x_{n-1}} & t_{n-1,1} \\
b_{n} e^{i \omega b_{n-1} x_{n-1}} & t_{n-1,2}
\end{array}\right| \frac{\operatorname{det} D_{n-1}(i \omega)}{\operatorname{det} D_{n}(i \omega)}+\left|\begin{array}{cc}
e^{-i \omega b_{n-1} x_{n-1}} & t_{n-1,1} \\
-b_{n} e^{-i \omega b_{n-1} x_{n-1}} & t_{n-1,2}
\end{array}\right| \frac{\operatorname{det} \tilde{D}_{n-1}(i \omega)}{\operatorname{det} D_{n}(i \omega)} \\
= & -e^{i \omega b_{n-1} x_{n-1}}\left|\begin{array}{ccc}
1 & 1 \\
b_{n} & b_{n-1}
\end{array}\right| t_{n-1,1} \frac{\operatorname{det} D_{n-1}(i \omega)}{\operatorname{det} D_{n}(i \omega)} \\
& +e^{-i \omega b_{n-1} x_{n-1}}\left|\begin{array}{cc}
1 & 1 \\
-b_{n} & b_{n-1}
\end{array}\right| t_{n-1,1} \frac{\left.\operatorname{det} \tilde{D}_{n-1}(i \omega)\right)}{\operatorname{det} D_{n}(i \omega)} \\
= & t_{n-1,1}\left(\left(b_{n-1}+b_{n}\right) e^{-i \omega b_{n-1} x_{n-1}} \frac{\left.\operatorname{det} \tilde{D}_{n-1}(i \omega)\right)}{\operatorname{det} D_{n}(i \omega)}+\left(b_{n}-b_{n-1}\right) e^{i \omega b_{n-1} x_{n-1}} \frac{\left.\operatorname{det} D_{n-1}(i \omega)\right)}{\operatorname{det} D_{n}(i \omega)}\right) \\
= & -t_{n-1,1} e^{-i \omega b_{n} x_{n-1}} \frac{\operatorname{det} \tilde{D}_{n}(i \omega)}{\operatorname{det} D_{n}(i \omega)},
\end{aligned}
$$

where the last identity involving $\operatorname{det} \tilde{D}_{n}, \operatorname{det} \tilde{D}_{n-1}$ and $\operatorname{det} D_{n-1}$ has been proved in [1, p. 871].

From (3.5) we have that

$$
i \omega t_{n-1,1}(i \omega)=\frac{b_{n}}{2} e^{-i \omega b_{n} x_{n-1}} \widehat{v_{0} I_{n}}\left(b_{n} \omega\right)
$$

and then we obtain the exact formula of $c_{2 n}(i \omega)$ in terms of $Q_{n}(i \omega)$ :

$$
i \omega c_{2 n}(i \omega)=-\frac{b_{n}}{2} e^{-2 i \omega b_{n} x_{n-1}} \frac{\operatorname{det} \tilde{D}_{n}(i \omega)}{\operatorname{det} D_{n}(i \omega)}=-\frac{b_{n}}{2} e^{-2 i \omega b_{n} x_{n-1}} Q_{n}(i \omega) \widehat{v_{0} I_{n}}\left(b_{n} \omega\right) .
$$

This identity is the key point in proving not only Theorem 1.1 but also Theorem 1.3.

It follows that

$$
\int_{\mathbb{R}}\left|\left(i \omega c_{2 n}(i \omega)\right)^{\vee}(x)\right| d x \leq \frac{b_{n}}{2}\left\|Q_{n}(i \omega)\right\|_{A P}\left\|v_{0}\right\|_{L^{1}(\mathbb{R})}
$$

and in view of Lemma 2.3 we have

$$
\int_{\mathbb{R}}\left|\left(i \omega c_{2 n}(i \omega)\right)^{\vee}(x)\right| d x \leq C\left(M, m, \tan \left(\sum_{k=1}^{n} \operatorname{arctanh}\left|d_{n}\right|\right)\right) .
$$

Thus by (2.21) and (2.22) the proof of the theorem in this case is finished. 
Case 2. Computing $c_{2 n}$ when supp $v_{0} \subset I_{1}$. Here all the terms $t_{k}, k=2, \ldots, n-1$ vanish and $t_{1,2}=-b_{2} t_{1,1}$. Hence

$$
c_{2 n}(i \omega)=\left(\operatorname{det} D_{n}(i \omega)\right)^{-1}\left|\begin{array}{cccccccc}
\mathbf{a}_{1} & B_{1} & 0 & 0 & 0 & 0 & 0 & t_{1} \\
0 & A_{2} & B_{2} & 0 & 0 & 0 & 0 & 0 \\
0 & 0 & A_{3} & B_{3} & 0 & 0 & 0 & 0 \\
- & - & - & - & - & - & - & - \\
0 & 0 & 0 & 0 & A_{n-3} & B_{n-3} & 0 & 0 \\
0 & 0 & 0 & 0 & 0 & A_{n-2} & B_{n-2} & 0 \\
0 & 0 & 0 & 0 & 0 & 0 & A_{n-1} & 0
\end{array}\right| .
$$

Developing the above determinant over blocks of two lines we obtain that

$$
c_{2 n}(i \omega)=\frac{\operatorname{det} A_{2} \cdots \operatorname{det} A_{n-1}}{\operatorname{det} D_{n}(i \omega)}\left|\begin{array}{cc}
e^{i \omega b_{1} x_{1}} & t_{1,1} \\
b_{2} e^{i \omega b_{1} x_{1}} & t_{1,2}
\end{array}\right|=\frac{\operatorname{det} A_{1} \cdots \operatorname{det} A_{n-1}}{\operatorname{det} D_{n}(i \omega)} e^{i \omega b_{1} x_{1}} t_{1,1}(i \omega) .
$$

Using estimate (3.7) on $t_{1,1}$ we get

$$
\int_{\mathbb{R}}\left|\left(i \omega c_{2 n}(i \omega)\right)^{\vee}(x)\right| d x \leq\left\|\frac{\operatorname{det} A_{1} \cdots \operatorname{det} A_{n-1}}{\operatorname{det} D_{n}(i \omega)}\right\|_{A P}\left\|v_{0}\right\|_{L^{1}(\mathbb{R})} .
$$

Since the proof of estimate (3.4) is the same as in Case 3 below (choose $k=1$ in (3.12)) we will skip it here.

Case 3. Computing $c_{2 n}$ when $\operatorname{supp} v_{0} \subset I_{k}, 2 \leq k \leq n-1$. Here $t_{k-1,2}=b_{k-1} t_{k-1,1}, t_{k, 2}=-b_{k+1} t_{k, 1}$, all the other terms in vector $T$ vanishing. Let us now compute $c_{2 n}$. It is given by

$$
\begin{aligned}
& c_{2 n}(i \omega)\left(\operatorname{det} D_{n}(i \omega)\right)=\left|\begin{array}{cccccccc}
a_{1} & B_{1} & 0 & 0 & 0 & 0 & 0 & 0 \\
0 & A_{2} & B_{2} & 0 & 0 & 0 & 0 & 0 \\
0 & 0 & A_{3} & B_{3} & 0 & 0 & 0 & 0 \\
- & - & - & - & - & - & - & - \\
- & - & - & A_{k-1} & B_{k-1} & - & - & t_{k-1} \\
0 & 0 & 0 & 0 & A_{k} & B_{k} & 0 & t_{k} \\
- & - & - & - & - & - & - & - \\
0 & 0 & 0 & 0 & 0 & A_{n-2} & B_{n-2} & 0 \\
0 & 0 & 0 & 0 & 0 & 0 & A_{n-1} & 0
\end{array}\right| \\
& =\left|\begin{array}{cccccccc}
a_{1} & B_{1} & 0 & 0 & 0 & 0 & 0 & 0 \\
0 & A_{2} & B_{2} & 0 & 0 & 0 & 0 & 0 \\
0 & 0 & A_{3} & B_{3} & 0 & 0 & 0 & 0 \\
- & - & - & - & - & - & - & - \\
- & - & - & A_{k-1} & B_{k-1} & - & - & t_{k-1} \\
0 & 0 & 0 & 0 & A_{k} & B_{k} & 0 & 0 \\
- & - & - & - & - & - & - & - \\
0 & 0 & 0 & 0 & 0 & A_{n-2} & B_{n-2} & 0 \\
0 & 0 & 0 & 0 & 0 & 0 & A_{n-1} & 0
\end{array}\right| \\
& +\left|\begin{array}{cccccccc}
a_{1} & B_{1} & 0 & 0 & 0 & 0 & 0 & 0 \\
0 & A_{2} & B_{2} & 0 & 0 & 0 & 0 & 0 \\
0 & 0 & A_{3} & B_{3} & 0 & 0 & 0 & 0 \\
- & - & - & - & - & - & - & - \\
- & - & - & A_{k-1} & B_{k-1} & - & - & 0 \\
0 & 0 & 0 & 0 & A_{k} & B_{k} & 0 & t_{k} \\
- & - & - & - & - & - & - & - \\
0 & 0 & 0 & 0 & 0 & A_{n-2} & B_{n-2} & 0 \\
0 & 0 & 0 & 0 & 0 & 0 & A_{n-1} & 0
\end{array}\right|
\end{aligned}
$$


Developing the determinants over blocks of two lines we find that

$$
\begin{aligned}
& c_{2 n}(i \omega)\left(\operatorname{det} D_{n}(i \omega)\right)=\operatorname{det} A_{k} \cdots \operatorname{det} A_{n-1}\left|\begin{array}{cccccccc}
a_{1} & B_{1} & 0 & 0 & 0 & 0 & 0 & 0 \\
0 & A_{2} & B_{2} & 0 & 0 & 0 & 0 & 0 \\
0 & 0 & A_{3} & B_{3} & 0 & 0 & 0 & 0 \\
- & - & - & - & - & - & - & - \\
0 & 0 & 0 & 0 & A_{k-3} & B_{k-3} & 0 & 0 \\
0 & 0 & 0 & 0 & 0 & A_{k-2} & B_{k-2} & 0 \\
0 & 0 & 0 & 0 & 0 & 0 & A_{k-1} & t_{k-1}
\end{array}\right| \\
& +\operatorname{det} A_{k+1} \cdots \operatorname{det} A_{n-1}\left|\begin{array}{cccccccc}
a_{1} & B_{1} & 0 & 0 & 0 & 0 & 0 & 0 \\
0 & A_{2} & B_{2} & 0 & 0 & 0 & 0 & 0 \\
0 & 0 & A_{3} & B_{3} & 0 & 0 & 0 & 0 \\
- & - & - & - & - & - & - & - \\
0 & 0 & 0 & 0 & A_{k-2} & B_{k-2} & 0 & 0 \\
0 & 0 & 0 & 0 & 0 & A_{k-1} & B_{k-1} & 0 \\
0 & 0 & 0 & 0 & 0 & 0 & A_{k} & t_{k}
\end{array}\right| .
\end{aligned}
$$

Since the components of $t_{k-1}$ satisfy $t_{k-1,2}=b_{k-1} t_{k-1,1}$ we can use the same argument as in Case 1 and we obtain that the first determinant equals

$$
-t_{k-1,1} e^{-i \omega b_{k} x_{k-1}} \operatorname{det} \tilde{D}_{k}(i \omega) \text {. }
$$

The second one could be computed in a similar way by expanding the determinant over the last two lines and using that

$$
\begin{aligned}
t_{k, 2} & =-b_{k+1} t_{k, 1}\left|\begin{array}{cccccccc}
a_{1} & B_{1} & 0 & 0 & 0 & 0 & 0 & 0 \\
0 & A_{2} & B_{2} & 0 & 0 & 0 & 0 & 0 \\
0 & 0 & A_{3} & B_{3} & 0 & 0 & 0 & 0 \\
- & - & - & - & - & - & - & - \\
0 & 0 & 0 & 0 & A_{k-2} & B_{k-2} & 0 & 0 \\
0 & 0 & 0 & 0 & 0 & A_{k-1} & B_{k-1} & 0 \\
0 & 0 & 0 & 0 & 0 & 0 & A_{k} & t_{k}
\end{array}\right| \\
& =-\left|\begin{array}{c}
e^{i \omega b_{k} x_{k}} \\
b_{k+1} e^{i \omega b_{k} x_{k}}-b_{k+1} t_{k, 1}
\end{array}\right| \operatorname{det} D_{k}(i \omega)+\left|\begin{array}{cc}
e^{-i \omega b_{k} x_{k}} \\
-b_{k+1} e^{-i \omega b_{k} x_{k}}-b_{k+1} t_{k, 1}
\end{array}\right| \operatorname{det} \tilde{D}_{k}(i \omega) \\
& =2 b_{k+1} t_{k, 1} \operatorname{det} D_{k}(i \omega) e^{i \omega b_{k} x_{k}}=-\operatorname{det}\left(A_{k}\right) t_{k, 1} \operatorname{det} D_{k}(i \omega) e^{i \omega b_{k} x_{k}} .
\end{aligned}
$$

This gives us that

$$
\begin{aligned}
c_{2 n}(i \omega) & =-\frac{\operatorname{det} A_{k} \cdots \operatorname{det} A_{n-1}}{\operatorname{det} D_{n}(i \omega)}\left(t_{k-1,1} e^{-i \omega b_{k} x_{k-1}} \operatorname{det} \tilde{D}_{k}(i \omega)+t_{k, 1} e^{i \omega b_{k} x_{k}} \operatorname{det} D_{k}(i \omega)\right) \\
& =-\frac{\operatorname{det} A_{k} \cdots \operatorname{det} A_{n-1} \operatorname{det} D_{k}(i \omega)}{\operatorname{det} D_{n}(i \omega)}\left(t_{k-1,1} e^{i \omega b_{k}\left(x_{k}-x_{k-1}\right)} Q_{k}(i \omega)+t_{k, 1}\right) e^{i \omega b_{k} x_{k}} .
\end{aligned}
$$

Applying estimate (3.7) for $t_{k, 1}$ and $t_{k-1,1}$ we obtain that

$$
\int_{\mathbb{R}}\left|\left(i \omega c_{2 n}(i \omega)\right)^{\vee}(t)\right| d t \leq C(m)\left(\left\|Q_{k}\right\|_{A P}+1\right)\left\|\frac{\operatorname{det} A_{k} \cdots \operatorname{det} A_{n-1} \operatorname{det} D_{k}(i \omega)}{\operatorname{det} D_{n}(i \omega)}\right\|_{A P}\left\|v_{0}\right\|_{L^{1}(\mathbb{R})} .
$$

Using now formula (2.12) that gives us the explicit expression of $\operatorname{det} D_{n}$ and $\operatorname{det} D_{k}$ we find that 


$$
\begin{aligned}
\frac{\operatorname{det} A_{k} \cdots \operatorname{det} A_{n-1} \operatorname{det} D_{k}(i \omega)}{\operatorname{det} D_{n}(i \omega)} & =\prod_{j=k}^{n-1} \frac{\operatorname{det} A_{j}}{b_{j}+b_{j+1}} \frac{e^{-i \omega\left(b_{j}-b_{j+1}\right) x_{j}}}{\left(1-d_{j} Q_{j}(i \omega)\right)} \\
& =(-1)^{n-k} \prod_{j=k}^{n-1} \frac{\left(1-d_{j}\right)}{\left(1-d_{j} Q_{j}(i \omega)\right)} e^{-i \omega\left(b_{j}-b_{j+1}\right) x_{j}} .
\end{aligned}
$$

Observe now that

$$
\frac{\left(1-d_{j}\right)}{\left(1-d_{j} Q_{j}(i \omega)\right)}=\left\{\begin{array}{rr}
\frac{1}{1+d_{j}}\left(1+d_{j} Q_{j+1}(i \omega) e^{2 i \omega b_{j+1}\left(x_{j+1}-x_{j}\right)}\right), & j=1, \ldots, n-2, \\
\frac{1}{1+d_{j}}\left(1+d_{j} Q_{j+1}(i \omega) e^{-2 i \omega b_{j+1} x_{j}}\right), & j=n-1 .
\end{array}\right.
$$

It gives us that for any $1 \leq j \leq n-1$ we have

$$
\left\|\frac{\left(1-d_{j}\right)}{\left(1-d_{j} Q_{j}(i \omega)\right)}\right\|_{A P} \leq \frac{1+\left|d_{j}\right|\left\|Q_{j+1}\right\|_{A P}}{1+d_{j}} \leq \frac{\exp \left(\left|d_{j}\right|\left\|Q_{j+1}\right\|_{A P}\right)}{1+d_{j}}
$$

and then

$$
\begin{aligned}
\left\|\prod_{j=k}^{n-1} \frac{\left(1-d_{j}\right)}{\left(1-d_{j} Q_{j}(i \omega)\right)}\right\|_{A P} & \leq \exp \left(\max _{j=k, \ldots, n-1}\left\|Q_{j+1}\right\|_{A P} \sum_{j=k}^{n-1}\left|d_{j}\right|\right) \cdot \prod_{j=k}^{n-1} \frac{1}{1+d_{j}} \\
& \leq \exp \left(c(m, M) \operatorname{Var}(a) \max _{j=k+1, \ldots, n}\left\|Q_{j}\right\|_{A P}\right) \cdot \prod_{j=k}^{n-1} \frac{1}{1+d_{j}} \\
& \leq \exp (c(m, M) \operatorname{Var}(a) \tan (\operatorname{Var}(\log (a)) / 4)) \cdot \prod_{j=k}^{n-1} \frac{1}{1+d_{j}} .
\end{aligned}
$$

The last term also satisfies

$$
\prod_{j=k}^{n-1} \frac{1}{1+d_{j}}=\prod_{j=k}^{n-1} \frac{b_{j}+b_{j+1}}{2 b_{j}}=\prod_{j=k}^{n-1}\left(1+\frac{\left|b_{j+1}-b_{j}\right|}{2 b_{j}}\right) \leq \exp (C(m, M) \operatorname{Var}(a)) .
$$

Putting now together estimates (3.12), (3.13), (3.14) and (3.15) we obtain that estimate (3.4) also holds in the case considered here.

Case 4. Prove (3.4) when supp $v_{0} \subset I_{k}, 2 \leq k \leq n-1$ and $k \leq j \leq n-1$. The previous cases prove (3.4) for $j=n$. Let us now prove that it holds for any $k \leq j \leq n-1$. We point out that once estimate (3.4) will be proved then it also holds for $1 \leq j \leq k-1$.

We now use that

$$
A_{n-1}\left(\begin{array}{c}
c_{2 n-3} \\
c_{2 n-2}
\end{array}\right)+\mathbf{b}_{n-1} c_{2 n}=\left(\begin{array}{l}
0 \\
0
\end{array}\right)
$$

and for $j \leq n-2$,

$$
A_{j}\left(\begin{array}{c}
c_{2 j-1} \\
c_{2 j}
\end{array}\right)+B_{j}\left(\begin{array}{l}
c_{2 j+1} \\
c_{2 j+2}
\end{array}\right)=\left(\begin{array}{l}
0 \\
0
\end{array}\right) .
$$

From identity (3.16) and the results of Case 3, we obtain that (3.4) holds for $j=n-1$ :

$$
\begin{aligned}
& \int_{\mathbb{R}}\left(\left|\left(i \omega c_{2 n-2}(i \omega)\right)^{\vee}(x)\right|+\left|\left(i \omega c_{2 n-3}(i \omega)\right)^{\vee}(x)\right|\right) d x \leq C(m, M) \int_{\mathbb{R}}\left|\left(i \omega c_{2 n}(i \omega)\right)^{\vee}(x)\right| d x \\
& \quad \leq C(m, M, \operatorname{Var}(a)) \int_{\mathbb{R}}\left|v_{0}(x)\right| d x .
\end{aligned}
$$

Applying identity (3.17) we obtain 


$$
\begin{aligned}
\left(\begin{array}{c}
c_{2 j-1} \\
c_{2 j}
\end{array}\right) & =-A_{j}^{-1} B_{j}\left(\begin{array}{l}
c_{2 j+1} \\
c_{2 j+2}
\end{array}\right) \\
& =\frac{1}{2 b_{j+1}}\left(\begin{array}{cc}
\left(b_{j+1}+b_{j}\right) e^{i \omega\left(b_{j+1}-b_{j}\right) x_{j}} & \left(b_{j+1}-b_{j}\right) e^{-i \omega\left(b_{j+1}+b_{j}\right) x_{j}} \\
\left(b_{j+1}-b_{j}\right) e^{i \omega\left(b_{j+1}+b_{j}\right) x_{j}} & \left(b_{j+1}+b_{j}\right) e^{-i \omega\left(b_{j+1}-b_{j}\right) x_{j}}
\end{array}\right)\left(\begin{array}{l}
c_{2 j+1} \\
c_{2 j+2}
\end{array}\right) .
\end{aligned}
$$

It implies that for $j \leq n-2$ we have

$$
\begin{aligned}
& \int_{\mathbb{R}}\left|\left(i \omega c_{2 j-1}(i \omega)\right)^{\vee}(t)\right|+\left|\left(i \omega c_{2 j}(i \omega)\right)^{\vee}(t)\right| d t \\
& \quad \leq \frac{\left|b_{j+1}-b_{j}\right|+b_{j}+b_{j+1}}{2 b_{j+1}} \int_{\mathbb{R}}\left|\left(i \omega c_{2 j+1}(i \omega)\right)^{\vee}(t)\right|+\left|\left(i \omega c_{2 j+2}(i \omega)\right)^{\vee}(t)\right| d t \\
& \quad \leq\left(1+\frac{\left|b_{j+1}-b_{j}\right|}{b_{j+1}}\right) \int_{\mathbb{R}}\left|\left(i \omega c_{2 j+1}(i \omega)\right)^{\vee}(t)\right|+\left|\left(i \omega c_{2 j+2}(i \omega)\right)^{\vee}(t)\right| d t .
\end{aligned}
$$

Using the same argument as in (3.15) we obtain that for any $k \leq j \leq n-1$

$$
\int_{\mathbb{R}}\left|\left(i \omega c_{2 j-1}(i \omega)\right)^{\vee}(t)\right|+\left|\left(i \omega c_{2 j}(i \omega)\right)^{\vee}(t)\right| d t \leq C(m, M, \operatorname{Var}(a)) \int_{\mathbb{R}}\left|v_{0}\right| .
$$

The proof of Theorem 1.1 is now complete.

Proof of Theorem 1.3. In this section we describe the manner in which the coefficient $a$ satisfying the conditions in Theorem 1.3 can be constructed. Without restricting the generality, we will construct a coefficient $a$ with $1 / 2 \leq a \leq 2$.

Let fix $d=\tanh ((\log 2) / 2)$. Since $\alpha \geq \pi / 2$, by Lemma 2.4, for any $N>0$ there exists a sequence $\left\{\tilde{d}_{k}\right\}_{k=1}^{n-1}$ such that

$$
\sum_{k=1}^{n-1} \operatorname{arctanh} \tilde{d}_{k}=\alpha, \quad 0 \leq \tilde{d}_{k} \leq d, \forall k=1, \ldots, n-1,
$$

and for any sequence of rationally independent numbers $\left\{c_{k}\right\}_{k=1}^{n}$ and any $\varepsilon_{k} \in\{ \pm 1\}, k=1, \ldots, n-1$, the sequence $\left\{Q_{k}\right\}_{k=1}^{n}$ associated to $\left\{c_{k}\right\}_{k=1}^{n-1}$ and $\left\{\varepsilon_{k} \tilde{d}_{k}\right\}_{k=1}^{n-1}$ satisfies $\left\|Q_{n}(i \omega)\right\|_{A P} \geq 2 N$.

Let us set $d_{k}=\varepsilon_{k} \tilde{d}_{k}=\varepsilon_{k}\left|d_{k}\right|$ where $\varepsilon_{k} \in\{ \pm 1\}$ will be chosen later. We now choose a sequence $\left\{b_{k}\right\}_{k=1}^{n}$ such that

$$
d_{k}=\varepsilon_{k}\left|d_{k}\right|=\frac{b_{k}-b_{k+1}}{b_{k}+b_{k+1}}<0, \quad k=1, \ldots, n-1 .
$$

This is possible since we can define recursively

$$
\frac{b_{k}}{b_{k+1}}=\exp \left(2 \operatorname{arctanh} d_{k}\right), k=1, \ldots, n-1, \quad b_{1}=1 .
$$

Let $a$ be the piecewise constant function defined by

$$
a(x)=b_{k}^{-2}, \quad x \in\left(x_{k-1}, x_{k}\right), \quad k=1, \ldots, n,
$$

where $x_{0}=-\infty, x_{n}=\infty$ and the points $\left\{x_{k}\right\}_{k=1}^{n-1}$ are chosen such that the numbers

$$
b_{k}\left(x_{k}-x_{k-1}\right), \quad k=2, \ldots, n-1
$$

are linearly independent over $\mathbb{Q}$. Using (2.21) we get that

$$
\operatorname{Var}(\log (a))=4 \sum_{k=1}^{n-1} \operatorname{arctanh}\left|d_{k}\right|=4 \alpha
$$


We now show how to choose the sequence $\left\{\varepsilon_{k}\right\}_{k=1}^{n-1}$ such that $a \in[1 / 2,2]$. Observe that for $x \in\left(x_{k-1}, x_{k}\right)$, the coefficient $a$ is given by

$$
a(x)=b_{k}^{-2}=\exp \left(2 \sum_{j=1}^{k-1} \operatorname{arctanh} d_{k}\right)=\exp \left(2 \sum_{j=1}^{k-1} \varepsilon_{k} \operatorname{arctanh}\left|d_{k}\right|\right) .
$$

Since $0 \leq \operatorname{arctanh}\left|d_{k}\right| \leq(\log 2) / 2$ we always can choose $\varepsilon_{k} \in\{ \pm\}$ such that

$$
\left|\sum_{j=1}^{k-1} \varepsilon_{j} \operatorname{arctanh}\right| d_{j}|| \leq \frac{\log 2}{2}, \quad \forall k=2, \ldots, n-1 .
$$

Indeed, for $k=2$ choose $\varepsilon_{1}=1$. Assume that the above inequality is true for some $k$. If $\sum_{j=1}^{k-1} \varepsilon_{j} \operatorname{arctanh}\left|d_{j}\right|$ belongs to $[0, \log (2) / 2]$ then choose $\varepsilon_{k}=-1$, otherwise $\varepsilon_{k}=1$. It follows that the above inequality will also hold for $k+1$. With this choice of $\left\{\varepsilon_{k}\right\}_{k=1}^{n-1}$ we obtain that coefficient $a$ satisfies $a \in[1 / 2,2]$.

Denoting $m=1 / 2$, in view of estimate (3.2) we have

$$
\frac{\sup _{x \in \mathbb{R}} \int_{\mathbb{R}}|v(t, x)| d t \quad \int_{\mathbb{R}}\left|i \omega c_{2 n}(i \omega)^{\vee}(t)\right| d t}{\left\|v_{0}\right\|_{L^{1}(\mathbb{R})}} \geq \frac{\left\|v_{0}\right\|_{L^{1}(\mathbb{R})}}{-2} \gtrsim \frac{\left.\|\left(Q_{n}(i \omega) \widehat{v}_{0}\right)^{\vee}\right) \|_{L^{1}(\mathbb{R})}}{\left\|v_{0}\right\|_{L^{1}(\mathbb{R})}}-m^{-2} .
$$

Let us now a sequence $v_{0 k}$ such that

$$
\frac{\left.\|\left(Q_{n}(i \omega) \widehat{v}_{0 k}\right)^{\vee}\right) \|_{L^{1}(\mathbb{R})}}{\left\|v_{0 k}\right\|_{L^{1}(\mathbb{R})}} \rightarrow\left\|Q_{n}\right\|_{A P} .
$$

This is always possible since $Q_{n}(i \omega)$ is an $L^{1}(\mathbb{R})$-Fourier multiplier and its norm is given by $\left\|Q_{n}\right\|_{A P}$, see [6, Th. 2.5.8, p. 141]. Thus we get for any $N$ large enough that

$$
\sup _{v_{0} \in L^{1}(\mathbb{R})} \frac{\sup _{x \in \mathbb{R}} \int_{\mathbb{R}}|v(t, x)| d t}{\left\|v_{0}\right\|_{L^{1}(\mathbb{R})}} \gtrsim\left\|Q_{n}\right\|_{A P}-m^{-2} \geq 2 N-4 \geq N .
$$

For the function $a$ chosen above we obtain that the left hand side of the above inequality can be arbitrarily large. The proof if now finished.

Proof of Theorem 1.2. We use formula (2.9) for the resolvent to find that for $x \in\left(x_{k-1}, x_{k}\right)$ solution $u$ of system (1.2) is given by

$$
\begin{aligned}
u(t, x)= & \frac{1}{i \pi} \int_{\mathbb{R}} e^{-i t \omega^{2}} \omega c_{2 k-1}(i \omega) e^{i \omega b_{k} x} d \omega+\frac{1}{i \pi} \int_{\mathbb{R}} e^{-i t \omega^{2}} \omega c_{2 k}(i \omega) e^{-i \omega b_{k} x} d \omega \\
& +\frac{b_{k}}{2 i \pi} \int_{\mathbb{R}} e^{-i t \omega^{2}} \omega \int_{I_{k}} u_{0}(y) e^{-i \omega_{k}|x-y|} d y d \omega .
\end{aligned}
$$

It follows that

$$
|u(t, x)| \leq \frac{C}{\sqrt{t}} \int_{\mathbb{R}}\left(\left|\left(i \omega c_{2 k-1}(i \omega)\right)^{\vee}(x)\right|+\left|\left(i \omega c_{2 k}(i \omega)\right)^{\vee}(x)\right|\right) d x+\frac{C}{\sqrt{t}}\left\|u_{0}\right\|_{L^{1}(\mathbb{R})} .
$$

Using now estimate (3.4) that has already been proved in the proof of Theorem 1.1 we obtain the desired estimate and the proof is finished.

\section{Proof of the two technical lemmas}

In this section we prove Lemma 2.3 and Lemma 2.4 using a fine analysis of the sequence $\left\{Q_{n}\right\}_{n \geq 1}$ defined by (2.16) by means of multi-variable series. 
4.1. The functions $\alpha(q), \beta(d), \gamma(t), E\left(d_{1}, \ldots, d_{n} ; q_{1}, \ldots, q_{n-1}\right)$ and $R\left(t_{1}, \ldots, t_{n} ; q_{1}, \ldots, q_{n-1}\right)$

We introduce the notations that will be used in this section. We denote by $\alpha(q)$ and $\beta(\sigma)$ the mappings:

$$
\alpha(q): z \mapsto q z, \quad \beta(\sigma): z \mapsto \frac{z+\sigma}{1+\sigma z} .
$$

Note that $q \mapsto \alpha(q)$ is a multiplicative mapping, i.e. $\alpha(q) \alpha\left(q^{\prime}\right)=\alpha\left(q q^{\prime}\right)$, and $\beta$ satisfies

$$
\beta(\sigma) \beta\left(\sigma^{\prime}\right)=\beta\left(\frac{\sigma+\sigma^{\prime}}{1+\sigma \sigma^{\prime}}\right) .
$$

From the formula

$$
\tanh (x+y)=\frac{\tanh x+\tanh y}{1+\tanh x \tanh y}
$$

we deduce that mapping $t \mapsto \gamma(t):=\beta(\tanh t)$ has the property

$$
\gamma(a) \gamma(b)=\gamma(a+b), \quad a, b \in \mathbb{R} .
$$

If $d=\left(d_{1}, \ldots, d_{n}\right)$, with $0 \leq d_{i}<1$ and $q=\left(q_{1}, \ldots, q_{n-1}\right)$ is a multivariable then we define the multivariable series

$$
E(d ; q):=\left(\beta\left(d_{n}\right) \alpha\left(q_{n-1}\right) \beta\left(d_{n-1}\right) \cdots \alpha\left(q_{1}\right) \beta\left(d_{1}\right)\right)(0) .
$$

With the convention that for $j=\left(j_{1}, \ldots, j_{n-1}\right)$ we write $q^{j}:=q_{1}^{j_{1}} \cdots q_{n-1}^{j_{n-1}}$. We introduce the norm $\|\cdot\|$ of a multivariable series by

$$
\left\|\sum_{j \in \mathbb{N}^{n-1}} a_{j} q^{j}\right\|=\sum_{j \in \mathbb{N}^{n-1}}\left|a_{j}\right| .
$$

It is easy to see that for any two series $\|a b\| \leq\|a\|\|b\|$.

Lemma 4.1. Let be $\left\{Q_{k}\right\}_{k \geq 1}$ defined by (2.16). Then for any $n \geq 1$

$$
\left\|Q_{n+1}\right\|_{A P} \leq\left\|E\left(\left|d_{1}\right|, \ldots,\left|d_{n}\right| ; q_{1}, \ldots, q_{n-1}\right)\right\|,
$$

with equality when $c_{1}, \ldots, c_{n-1}$ are linearly independent over $\mathbb{Q}$.

Proof. We write $d_{k}=\varepsilon_{k}\left|d_{k}\right|$ with $\varepsilon_{k}= \pm 1$. The sequence $\left\{Q_{k}\right\}_{k \geq 1}$ can be written as

$$
\begin{aligned}
Q_{k}(i \omega) & =-\varepsilon_{k-1} e^{i \omega c_{k-1}} \frac{-\varepsilon_{k-1} Q_{k-1}(i \omega)+\left|d_{k-1}\right|}{1-\varepsilon_{k-1}\left|d_{k-1}\right| Q_{k-1}(i \omega)} \\
& =\left(\alpha\left(-\varepsilon_{k-1} e^{i \omega c_{k-1}}\right) \beta\left(\left|d_{k-1}\right|\right) \alpha\left(-\varepsilon_{k-1}\right)\right)\left(Q_{k-1}(i \omega)\right), k \geq 2 .
\end{aligned}
$$

It follows that

$$
Q_{n+1}(i \omega)=\left(\alpha\left(-\varepsilon_{n} e^{i \omega c_{n}}\right) \beta\left(\left|d_{n}\right|\right) \alpha\left(\varepsilon_{n-1} \varepsilon_{n} e^{i \omega c_{n-1}}\right) \beta\left(\left|d_{n-1}\right|\right) \cdots \alpha\left(\varepsilon_{1} \varepsilon_{2} e^{i \omega c_{1}}\right) \beta\left(\left|d_{1}\right|\right)\right)(0) .
$$

With the above notations it follows that

$$
Q_{n+1}(i \omega)=-\varepsilon_{n} e^{i \omega c_{n}} E\left(\left|d_{1}\right|, \ldots,\left|d_{n}\right| ; \varepsilon_{1} \varepsilon_{2} e^{i \omega c_{1}}, \ldots, \varepsilon_{n-1} \varepsilon_{n} e^{i \omega c_{n-1}}\right) .
$$

Since $\left|-\varepsilon_{n}\right|=1$ and $\left|\varepsilon_{k} \varepsilon_{k+1}\right|=1$ for $1 \leq k \leq n-1$ we have

$$
\left\|Q_{n+1}\right\|_{A P} \leq\left\|E\left(\left|d_{1}\right|, \ldots,\left|d_{n}\right| ; q_{1}, \ldots, q_{n-1}\right)\right\|,
$$

with equality when $c_{1}, \ldots, c_{n-1}$ are linearly independent over $\mathbb{Q}$.

In the following we will estimate in a clever way the norm of $E\left(\left|d_{1}\right|, \ldots,\left|d_{n}\right| ; q_{1}, \ldots, q_{n-1}\right)$. For any $t=$ $\left(t_{1}, \ldots, t_{n}\right)$ we define the series $R(t ; q)$ in the multivariable $q=\left(q_{1}, \ldots, q_{n-1}\right)$ by 


$$
\begin{aligned}
R(t ; q) & =\left(\gamma\left(t_{n}\right) \alpha\left(q_{n-1}\right) \gamma\left(t_{n-1}\right) \cdots \alpha\left(q_{1}\right) \gamma\left(t_{1}\right)\right)(0) \\
& =E\left(\tanh t_{1}, \ldots, \tanh t_{n} ; q\right) .
\end{aligned}
$$

When there is no risk of confusion we will write only $R(t)$.

The fact that tanh gives a bijection between $[0, \infty)$ and $[0,1)$ allows us to find estimates for $\| E\left(d_{1}, \ldots, d_{n}\right.$; $\left.q_{1}, \ldots, q_{n-1}\right) \|$ in terms of estimates for $\left\|R\left(t_{1}, \ldots, t_{n} ; q_{1}, \ldots, q_{n-1}\right)\right\|$. Dealing with $R$ rather than $E$ is more advantageous because it allows us to use the formula $\gamma(a) \gamma(b)=\gamma(a+b)$.

\subsection{Estimates on partitions}

For any positive number $x$ we consider the set of all partitions of $x$ :

$$
A_{x}=\left\{t=\left(t_{1}, \ldots, t_{n}\right) \mid t_{j}>0, \sum_{j=1}^{n} t_{j}=x\right\} .
$$

If $t=\left(t_{1}, \ldots, t_{n}\right) \in A_{x}$ the sequence of the partial sums $x_{k}=\sum_{j=1}^{k} t_{j}, k=0, \ldots, n$ is a partition of the interval $[0, x]$, i.e. we have $0=x_{0}<x_{1}<\ldots<x_{n}=x$. We get a one-to-one correspondence between $A_{x}$ and the partitions of [0,x].

If $t=\left(t_{1}, \ldots, t_{n}\right)$ and $s=\left(s_{1}, \ldots, s_{m}\right)$ are two elements in $A_{x}$ we say that $s$ is finer than $t$ and we write $t \prec s$ if the sequence of partial sums of $s$ contains the sequence of partial sums of $t$, i.e. there is a sequence $1 \leq k_{1}<\ldots<k_{n}=m$ such that

$$
\sum_{j=1}^{l} t_{j}=\sum_{j=1}^{k_{l}} s_{j}, \quad \forall 1 \leq l \leq n .
$$

It follows that $\left(A_{x}, \prec\right)$ is a directed set.

Lemma 4.2. Let be $0 \leq d_{j} \leq 1,1 \leq j \leq n$ and $P=\prod_{j=1}^{n}\left(1+d_{j}\right), p=\prod_{j=1}^{n}\left(1-d_{j}\right)$. Then the map

$$
\beta\left(d_{n}\right) \alpha\left(q_{n-1}\right) \cdots \beta\left(d_{1}\right) \alpha\left(q_{0}\right)
$$

is given by the map $z \mapsto(a z+b) /(c z+d)$, where $a, b, c, d \in \mathbb{R}\left[q_{0}, \ldots, q_{n-1}\right]$ have non-negative coefficients,

$$
\|a\|=\|c\|=\frac{1}{2}(P+p),\|b\|=\|d\|=\frac{1}{2}(P-p),
$$

the coefficient of $q_{0} \cdots q_{n-1}$ in polynomial $a$ and the constant term in polynomial $d$ is 1 .

Proof. Note that

$$
\frac{1}{2}(P+p)=\sum_{k \text { even }} \sum_{1 \leq i_{1}<\ldots<i_{k} \leq n} d_{i_{1}} \cdots d_{i_{k}}
$$

and

$$
\frac{1}{2}(P-p)=\sum_{k \text { odd }} \sum_{1 \leq i_{1}<\ldots<i_{k} \leq n} d_{i_{1}} \cdots d_{i_{k}} .
$$

Let $M\left(q_{0}, \ldots, q_{n-1}\right)$ be the matrix

$$
M\left(q_{0}, \ldots, q_{n-1}\right)=\left(\begin{array}{ll}
a & b \\
c & d
\end{array}\right)=\left(\begin{array}{cc}
1 & d_{n} \\
d_{n} & 1
\end{array}\right)\left(\begin{array}{cc}
q_{n-1} & 0 \\
0 & 1
\end{array}\right) \cdots\left(\begin{array}{cc}
1 & d_{1} \\
d_{1} & 1
\end{array}\right)\left(\begin{array}{cc}
q_{0} & 0 \\
0 & 1
\end{array}\right) .
$$

Thus the map $\beta\left(d_{n}\right) \alpha\left(q_{n-1}\right) \cdots \beta\left(d_{1}\right) \alpha\left(q_{0}\right)$ is given by

$$
z \mapsto \frac{a z+b}{c z+d} .
$$

Since $d_{j}, j \geq 1$ are nonnegative, $a, b, c, d \in R\left[q_{0}, \ldots, q_{n-1}\right]$ are polynomials with non-negative coefficients. Therefore $\|a\|=a(1, \ldots, 1)$ and similarly for $b, c, d$. 
Denoting

$$
A=\left(\begin{array}{ll}
0 & 1 \\
1 & 0
\end{array}\right), B_{0}=\left(\begin{array}{ll}
0 & 0 \\
0 & 1
\end{array}\right), B_{1}=\left(\begin{array}{ll}
1 & 0 \\
0 & 0
\end{array}\right)
$$

we have that

$$
M\left(q_{0}, \ldots, q_{n-1}\right)=\left(I+d_{n} A\right)\left(B_{0}+q_{n-1} B_{1}\right) \cdots\left(I+d_{1} A\right)\left(B_{0}+q_{0} B_{1}\right) .
$$

Since $B_{0}+B_{1}=I$ and $A^{2}=I$, it follows that

$$
\begin{aligned}
M(1, \ldots, 1) & =\left(I+d_{n} A\right) \cdots\left(I+d_{1} A\right)=\sum_{k=0}^{n} \sum_{\begin{array}{l}
1 \leq i_{1}<\ldots<i_{k} \leq n \\
d_{1}
\end{array}} d_{i_{1}} \cdots d_{i_{k}} A^{k} \\
& =\left(\sum_{k \text { even } 1 \leq i_{1}<\ldots<i_{k} \leq n} d_{i_{1}} \cdots d_{i_{k}}\right) I+\left(\sum_{k \text { odd }} \sum_{\left.\begin{array}{l}
1 \leq i_{1}<\ldots<i_{k} \leq n \\
\frac{1}{2}(P-p) \\
\frac{1}{2}(P+p)
\end{array}\right) .} d_{i_{1}} \cdots d_{i_{k}}\right) A \\
& =\frac{1}{2}(P+p) I+\frac{1}{2}(P-p) A=\left(\begin{array}{c}
\frac{1}{2}(P+p) \\
\frac{1}{2}(P-p)
\end{array}\right.
\end{aligned}
$$

It implies that

$$
a(1, \ldots, 1)=c(1, \ldots, 1)=\frac{1}{2}(P+p)
$$

and

$$
b(1, \ldots, 1)=d(1, \ldots, 1)=\frac{1}{2}(P-p) .
$$

Then the first part of Lemma 4.2 is proved.

Let us now compute the coefficient of $q_{0} \cdots q_{n-1}$ in polynomial $a$ and the constant term in polynomial $d$. In view of (4.21) we have

$$
M\left(q_{0}, \ldots, q_{n-1}\right)=\sum_{\left(k_{0}, \ldots, k_{n-1}\right) \in\{0,1\}^{n}} q_{0}^{k_{0}} \cdots q_{n-1}^{k_{n-1}} M_{k_{0}, \ldots, k_{n-1}},
$$

where

$$
M_{k_{0}, \ldots, k_{n-1}}=\left(I+d_{n} A\right) B_{k_{n-1}} \cdots\left(I+d_{1} A\right) B_{k_{0}} .
$$

To determine the coefficient of $q_{0} \cdots q_{n-1}$ and the constant term of $d$ one has to calculate $M_{1, \ldots, 1}$ and $M_{0, \ldots, 0}$, respectively.

Note that $B_{0}^{2}=B_{0}, B_{1}^{2}=B_{1}$ and $B_{0} A B_{0}=B_{1} A B_{1}=0$ so $B_{0}\left(I+d_{j} A\right) B_{0}=B_{0}$ and $B_{1}\left(I+d_{j} A\right) B_{1}=B_{1}$. Hence

$$
M_{0 \ldots, 0}=\left(I+d_{n} A\right) B_{0} \cdots\left(I+d_{1} A\right) B_{0}=\left(I+d_{n} A\right) B_{0}=\left(\begin{array}{cc}
0 & d_{n} \\
0 & 1
\end{array}\right)
$$

so the constant term of $d$ is one. Also

$$
M_{1, \ldots, 1}=\left(I+d_{n} A\right) B_{1} \cdots\left(I+d_{1} A\right) B_{1}=\left(I+d_{n} A\right) B_{1}=\left(\begin{array}{cc}
1 & 0 \\
d_{n} & 0
\end{array}\right)
$$

so the coefficient of $q_{1} \cdots q_{n-1}$ in $a$ is one.

Corollary 4.1. Let $\left(t_{1}, \ldots, t_{n}\right) \in A_{x}$. The map

$$
\gamma\left(t_{n}\right) \alpha\left(q_{n-1}\right) \cdots \gamma\left(t_{1}\right) \alpha\left(q_{0}\right)
$$

is given by $z \mapsto(a z+b) /(c z+d)$, where $a, b, c, d \in \mathbb{R}\left[q_{0}, \ldots, q_{n-1}\right]$ have non-negative coefficients,

$$
\|a\|=\|d\|<\cosh x,\|b\|=\|c\|<\sinh x,
$$

the coefficient of $q_{0} \cdots q_{n-1}$ in $a$ and the constant term of $d$ is 1 . 
Proof. Since $\gamma(t)=\beta(\tanh t)$ we can use Lemma 4.2 with $d_{j}=\tanh t_{j}$. Using the notations of Lemma 4.2, we must prove that

$$
\frac{P+p}{2}<\cosh x \text { and } \frac{P-p}{2}<\sinh x .
$$

Since

$$
1+d_{j}=1+\tanh t_{j}=\frac{e^{t_{j}}}{\cosh t_{j}}, \quad 1-d_{j}=1-\tanh t_{j}=\frac{e^{-t_{j}}}{\cosh t_{j}}
$$

we get

$$
P=e^{x}\left(\prod_{j} \cosh t_{j}\right)^{-1}<e^{x}, p=e^{-x}\left(\prod_{j} \cosh t_{j}\right)^{-1}<e^{-x} .
$$

Thus (4.22) immediately follows.

Lemma 4.3. For any two partitions $s, t \in A_{x}$ with $t \prec s$ the following holds for any positive integer $r$

$$
\left\|R(t)^{r}\right\| \leq\left\|R(s)^{r}\right\| .
$$

Proof. Let $t=\left(t_{1}, \ldots, t_{n}\right) \prec s=\left(s_{1}, \ldots, s_{m}\right)$ be two elements in $A_{x}$. In order to prove this result we relate the two series $R(t)$ and $R(s)$. Observe that $R(t)$ and $R(s)$ depend on $n-1$ and $m-1$ variables respectively, $m \geq n$. Since $t \prec s$ there are $0=k_{0}<k_{1}<\cdots<k_{n}=m$ such that

$$
t_{l}=\sum_{j=k_{l-1}+1}^{k_{l}} s_{j}, \quad \forall 1 \leq l \leq n .
$$

Using that $\gamma(a+b)=\gamma(a) \gamma(b)$ it follows that

$$
\gamma\left(t_{l}\right)=\prod_{j=k_{l-1}+1}^{k_{l}} \gamma\left(s_{j}\right)
$$

Since $\alpha(1)=1$ we also have

$$
\gamma\left(t_{l}\right)=\gamma\left(s_{k_{l-1}+1}\right) \alpha(1) \gamma\left(s_{k_{l-1}+2}\right) \alpha(1) \cdots \alpha(1) \gamma\left(s_{k_{l}}\right) .
$$

Replacing $\gamma\left(t_{l}\right)$ by the above formula in the definition of $R(t)$, (4.20), one gets

$$
\begin{aligned}
R\left(t ; q_{1}, \ldots, q_{n-1}\right) & =\left(\gamma\left(t_{n}\right) \alpha\left(q_{n-1}\right) \gamma\left(t_{n-1}\right) \cdots \alpha\left(q_{1}\right) \gamma\left(t_{1}\right)\right)(0) \\
& =R\left(s ; 1, \ldots, 1, q_{1}, 1, \ldots, 1, q_{2}, \ldots, q_{n-1}, 1 \ldots, 1\right),
\end{aligned}
$$

where the blocks of one above have lengths $k_{n}-k_{n-1}-1, \ldots, k_{2}-k_{1}-1, k_{1}-k_{0}-1$. Thus $\|R(t)\| \leq\|R(s)\|$. For $r \geq 2$ the argument is similar since

$$
R\left(t ; q_{1}, \ldots, q_{n-1}\right)^{r}=R\left(s ; 1, \ldots, 1, q_{1}, \ldots, q_{n-1}, 1 \ldots, 1\right)^{r} .
$$

The proof is finished.

\subsection{Upper bounds for $R(t)$}

We now obtain some properties of the multivariable series $R(t)$ introduced above. For any integer $r \geq 0$ we define the function $f_{r}:(0, \infty) \rightarrow(0, \infty]$ by

$$
f_{r}(x)=\sup _{t \in A_{x}}\left\|R(t)^{r}\right\| \text {. }
$$

In particular, $f_{0} \equiv 1$. We note that $f_{r_{1}+r_{2}}(x) \leq f_{r_{1}}(x) f_{r_{2}}(x)$. In particular for any integer $r \geq 1, f_{r}(x) \leq f_{1}(x)^{r}$. 
The first estimate for $f_{1}$ is given in the following lemma.

Lemma 4.4. For any $x \in(0, \log (2+\sqrt{3}))$ the following holds

$$
f_{1}(x) \leq \frac{\sinh x}{2-\cosh x}
$$

Proof. Let us choose $t=\left(t_{1}, \ldots, t_{n}\right) \in A_{x}$ and denote $q=\left(q_{1}, \ldots, q_{n-1}\right)$. We will show that

$$
\|R(t ; q)\| \leq \frac{\sinh x}{2-\cosh x} .
$$

By definition

$$
R(t ; q)=\left(\gamma\left(t_{n}\right) \alpha\left(q_{n-1}\right) \gamma\left(t_{n-1}\right) \cdots \alpha\left(q_{1}\right) \gamma\left(t_{1}\right)\right)(0) .
$$

From Corollary 4.1 it follows that

$$
\left(\gamma\left(t_{n}\right) \alpha\left(q_{n-1}\right) \gamma\left(t_{n-1}\right) \cdots \alpha\left(q_{1}\right) \gamma\left(t_{1}\right) \alpha\left(q_{0}\right)\right)(z)=\frac{a z+b}{c z+d} .
$$

Since $\alpha\left(q_{0}\right)(0)=0$ when we take $z=0$ in the above equation we get

$$
R(t ; q)=\frac{b}{d}
$$

Using Corollary 4.1, we have that

$$
\|b\|<\sinh x \text { and }\|d-1\|=\|d\|-1<\cosh x-1 .
$$

Then, for any $x$ satisfying $\cosh x<2$ the following holds

$$
\|R(t ; q)\| \leq \frac{\|b\|}{1-\|1-d\|} \leq \frac{\sinh x}{2-\cosh x} .
$$

(Note that $a, b, c, d \in \mathbb{R}\left[q_{0}, q_{1}, \ldots, q_{n-1}\right]$ but the variable $q_{0}$ is superfluous in $\frac{b}{d}=R(t ; q)$. It does not appear effectively.)

Since the partition $t \in A_{x}$ has been arbitrarily chosen we obtain that estimate (4.23) holds for all $x<\operatorname{arccosh}(2)=$ $\log (2+\sqrt{3})$.

Lemma 4.5. For any positive integer $r$ the function $f_{r}$ is increasing.

Proof. Let us observe that for any $d \in[0,1)$ we have $\beta(d) \alpha(-1) \beta(d) \alpha(-1)=1$. Thus, for any nonnegative $t, \gamma(t)$ satisfies $\gamma(t) \alpha(-1) \gamma(t) \alpha(-1)=1$.

Let us now choose $0<x<y$, where $y=x+z$ with $z>0$. Let $t=\left(t_{1}, \ldots, t_{n}\right) \in A_{x}$. Then $s:=\left(t_{1}, \ldots, t_{n}\right.$, $z / 2, z / 2) \in A_{y}$. Since $\gamma(z / 2) \alpha(-1) \gamma(z / 2) \alpha(-1)=1$ we have:

$$
\begin{aligned}
R\left(t ; q_{1}, \ldots, q_{n-1}\right) & =\left(\gamma\left(\frac{z}{2}\right) \alpha(-1) \gamma\left(\frac{z}{2}\right) \alpha(-1) \gamma\left(t_{n}\right) \alpha\left(q_{n-1}\right) \cdots \alpha\left(q_{1}\right) \gamma\left(q_{1}\right)\right)(0) \\
& =R\left(s ;\left(q_{1}, \ldots, q_{n-1},-1,-1\right)\right)
\end{aligned}
$$

Hence for any integer $r \geq 1$,

$$
R\left(t ; q_{1}, \ldots, q_{n-1}\right)^{r}=R\left(s ; q_{1}, \ldots, q_{n-1},-1,-1\right)^{r},
$$

which implies that $\left\|R(t)^{r}\right\| \leq\left\|R(s)^{r}\right\|$. This implies that for any $t \in A_{x}$ there is some $s \in A_{y}$ with $\left\|R(t)^{r}\right\| \leq\left\|R(s)^{r}\right\|$. It follows that $f_{r}(x) \leq f_{r}(y)$ so $f_{r}$ is an increasing function.

We denote $I=\left\{x \in(0, \infty) \mid f_{1}(x)<\infty\right\}$. In view of Lemma 4.4 and Lemma 4.5, the set $I$ is an interval that includes $(0, \log (2+\sqrt{3}))$. Moreover, all the functions $f_{r}, r \geq 1$, are finite on interval $I$ since $f_{r}(x) \leq f_{1}^{r}(x)$. Now we prove that $f_{r}$ are differentiable. 
Lemma 4.6. The set $I$ is an open interval. For any integer $r \geq 1$, function $f_{r}$ is differentiable on $I$ and satisfies $f_{r}^{\prime}=r\left(f_{r-1}+f_{r+1}\right)$.

Proof. Let $\varepsilon$ and $x$ be positive numbers. For any partition $s \in A_{x+\varepsilon}$ there is a finer partition, $\tilde{s}$, of the form $\tilde{s}=\left(t, t^{\prime}\right) \in$ $A_{x} \times A_{\varepsilon}$ (because for any partition of $[0, x+\varepsilon]$ there is a finer one containing $x$ ). Then

$$
\|R(s)\| \leq\|R(\tilde{s})\| \leq f_{1}(x+\varepsilon)
$$

and so

$$
f_{1}(x+\varepsilon)=\sup _{\left(t, t^{\prime}\right) \in A_{x} \times A_{\varepsilon}}\left\|R\left(t, t^{\prime}\right)\right\| .
$$

Let us consider $\left(t, t^{\prime}\right) \in A_{x} \times A_{x}, t=\left(t_{1}, \ldots, t_{n}\right), t^{\prime}=\left(t_{n+1}, \ldots, t_{m}\right)$. Denoting $q=\left(q_{1}, \ldots, q_{n-1}\right)$ and $q^{\prime}=$ $\left(q_{n}, \ldots, q_{m-1}\right)$ we obtain that

$$
R\left(t, t^{\prime} ; q, q^{\prime}\right)=\left(\gamma\left(t_{m}\right) \alpha\left(q_{m-1}\right) \cdots \gamma\left(t_{n+1}\right) \alpha\left(q_{n}\right)\right)(R(t ; q)) .
$$

Since $t \in A_{\varepsilon}$ by Corollary 4.1 we have that

$$
\gamma\left(t_{m}\right) \alpha\left(q_{m-1}\right) \cdots \gamma\left(t_{n+1}\right) \alpha\left(q_{n}\right)
$$

is given by $z \mapsto \frac{a z+b}{c z+d}$, where $a, b, c, d \in \mathbb{R}\left[q_{n}, \ldots, q_{m-1}\right]$ have non-negative coefficients, $\|a\|=\|d\|<\cosh \varepsilon,\|b\|=$ $\|c\|<\sinh \varepsilon$, the coefficient of $q_{n} \cdots q_{m-1}$ in $a$ and the constant term of $d$ are 1 . Then $\|a\|=1+\left\|a-q_{n} \cdots q_{m-1}\right\|$ and $\|d\|=1+\|d-1\|$ so

$$
\left\|a-q_{n} \cdots q_{m-1}\right\|=\|a\|-1=\|d\|-1=\|d-1\|<\cosh \varepsilon-1 .
$$

Then

$$
R\left(t, t^{\prime}\right)=\frac{a R(t)+b}{c R(t)+d}=\frac{a R(t)+b}{1+(c R(t)+d-1)} .
$$

Since $t \in A_{x}$ we have $\|R(t)\| \leq f_{1}(x)<\infty$. We also have $\cosh \varepsilon=1+O\left(\varepsilon^{2}\right)$ and $\sinh \varepsilon=O(\varepsilon)$ so

$$
a R(t)+b=q_{n} \cdots q_{m-1} R(t)+\left(a-q_{n} \cdots q_{m-1}\right) R(t)+b=q_{n} \cdots q_{m-1} R(t)+b+O\left(\varepsilon^{2}\right)
$$

and

$$
c R(t)+d=1+c R(t)+(d-1)=1+c R(t)+O\left(\varepsilon^{2}\right) .
$$

Then for $\varepsilon<\varepsilon_{0}$, $\varepsilon_{0}$ small enough depending on $f_{1}(x)$, the following holds

$$
\begin{aligned}
R\left(t, t^{\prime}\right) & =\left(q_{n} \cdots q_{m-1} R(t)+b+O\left(\varepsilon^{2}\right)\right)\left(1-c R(t)+O\left(\varepsilon^{2}\right)\right) \\
& =q_{n} \cdots q_{m-1} R(t)+b-q_{n} \cdots q_{m-1} c R(t)^{2}+O\left(\varepsilon^{2}\right) .
\end{aligned}
$$

Since $b-q_{n} \cdots q_{m-1} c R(t)=O(\varepsilon)$ this implies that for any $r \geq 1$ we have

$$
\begin{aligned}
R\left(t, t^{\prime}\right)^{r} & =\left(q_{n} \cdots q_{m-1}\right)^{r} R(t)^{r}+r\left(q_{n} \cdots q_{m-1}\right)^{r-1} R(t)^{r-1}\left(b-q_{n} \cdots q_{m-1} c R(t)^{2}\right)+O\left(\varepsilon^{2}\right) \\
& =\left(q_{n} \cdots q_{m-1}\right)^{r} R(t)^{r}+r\left(q_{n} \cdots q_{m-1}\right)^{r-1}\left(b R(t)^{r-1}-q_{n} \cdots q_{m-1} c R(t)^{r+1}\right)+O\left(\varepsilon^{2}\right) .
\end{aligned}
$$

Using that $\sinh \varepsilon=\varepsilon+O\left(\varepsilon^{2}\right)$ it follows that

$$
\begin{aligned}
\left\|R\left(t, t^{\prime}\right)^{r}\right\| & \leq\left\|R(t)^{r}\right\|+r\left\|R(t)^{r-1}\right\| \cdot\|b\|+r\left\|R(t)^{r+1}\right\| \cdot\|c\|+O\left(\varepsilon^{2}\right) \\
& \leq f_{r}(x)+r f_{r-1}(x) \sinh \varepsilon+r f_{r+1}(x) \sinh \varepsilon+O\left(\varepsilon^{2}\right) \\
& =f_{r}(x)+r\left(f_{r-1}(x)+f_{r+1}(x)\right) \varepsilon+O\left(\varepsilon^{2}\right) .
\end{aligned}
$$

Thus, for $\varepsilon$ small enough we have

$$
f_{r}(x+\varepsilon) \leq f_{r}(x)+r\left(f_{r-1}(x)+f_{r+1}(x)\right) \varepsilon+O\left(\varepsilon^{2}\right) .
$$

This implies that $I$ is an open interval. 
For the reverse inequality we will take $m=n+1$ so $t^{\prime}$ has dimension one, $t^{\prime}=(\varepsilon)$, and $q^{\prime}=q_{n}$. Then $(t, \varepsilon) \in A_{x+\varepsilon}$ so $f_{r}(x+\varepsilon) \geq\left\|R(t, \varepsilon)^{r}\right\|$. We have $R\left(t, \varepsilon ; q, q_{n}\right)=\gamma(\varepsilon) \alpha\left(q_{n}\right)(R(t, q))$, i.e.

$$
R(t, \varepsilon)=\frac{q_{n} R(t)+\tanh \varepsilon}{q_{n} \tanh \varepsilon R(t)+1} .
$$

Since $\tanh \varepsilon=\varepsilon+O\left(\varepsilon^{2}\right)$ by same argument as above we prove that

$$
R(t, \varepsilon)=q_{n} R(t)+\varepsilon\left(1-q_{n}^{2} R(t)^{2}\right)+O\left(\varepsilon^{2}\right)
$$

and, more generally,

$$
R(t, \varepsilon)^{r}=q_{n}^{r} R(t)^{r}+r \varepsilon q_{n}^{r-1}\left(R(t)^{r-1}-q_{n}^{2} R(t)^{r+1}\right)+O\left(\varepsilon^{2}\right) .
$$

Since $R(t)^{r-1}, R(t)^{r}, R(t)^{r+1} \in \mathbb{R}\left[\left[q_{0}, \ldots, q_{n-1}\right]\right]$ it follows that

$$
\left\|q_{n}^{r} R(t)^{r}+r \varepsilon\left(q_{n}^{r-1} R(t)^{r-1}-q_{n}^{r+1} R(t)^{r+1}\right)\right\|=\left\|R(t)^{r}\right\|+r \varepsilon\left\|R(t)^{r-1}\right\|+r \varepsilon\left\|R(t)^{r+1}\right\| .
$$

Hence for any $t \in A_{x}$ and $\varepsilon$ small enough

$$
f_{r}(x+\varepsilon) \geq\left\|R(t, \varepsilon)^{r}\right\| \geq\left\|R(t)^{r}\right\|+r \varepsilon\left(\left\|R(t)^{r-1}\right\|+\left\|R(t)^{r+1}\right\|\right)+O\left(\varepsilon^{2}\right)
$$

and then

$$
f_{r}(x+\varepsilon) \geq f_{r}(x)+\varepsilon r\left(f_{r-1}(x)+f_{r+1}(x)\right)+O\left(\varepsilon^{2}\right) .
$$

Using (4.24) and (4.25) we obtain that $f_{r}$ satisfies

$$
f_{r}(x+\varepsilon)=f_{r}(x)+\varepsilon r\left(f_{r-1}(x)+f_{r+1}(x)\right)+O\left(\varepsilon^{2}\right) .
$$

Thus function $f_{r}$ is right differentiable and satisfies

$$
\lim _{e \searrow 0} \frac{f_{r}(x+\varepsilon)-f_{r}(x)}{\varepsilon}=r\left(f_{r-1}(x)+f_{r+1}(x)\right) .
$$

Moreover, since $f_{k}(x-\varepsilon) \leq f_{k}(x) \leq f_{1}^{k}(x), k \in\{r-1, r+1\}$, by applying the same argument as in the proof of (4.24) and (4.25) to $x^{\prime}=x-\varepsilon$ we obtain that

$$
f_{r}(x)=f_{r}(x-\varepsilon)+\varepsilon r\left(f_{r-1}(x-\varepsilon)+f_{r+1}(x-\varepsilon)\right)+O\left(\varepsilon^{2}\right)=f_{r}(x-\varepsilon)+O(\varepsilon)
$$

which proves that $f_{r}$ is also left continuous.

For the left derivative of $f_{r}$ at $x$ we apply the previous analysis to the point $x^{\prime}=x-\varepsilon$

$$
\frac{f_{r}(x)-f_{r}(x-\varepsilon)}{\varepsilon}=r\left(f_{r-1}(x-\varepsilon)+f_{r+1}(x-\varepsilon)\right)+O(\varepsilon) .
$$

Since $f_{r-1}, f_{r+1}$ are continuous we obtain

$$
\lim _{\varepsilon \searrow 0} \frac{f_{r}(x)-f_{r}(x-\varepsilon)}{\varepsilon}=r\left(f_{r-1}(x)+f_{r+1}(x)\right) .
$$

The proof of Lemma 4.6 is now finished.

Theorem 4.1. We have $I=(0, \pi / 2)$ and for any $r \geq 1$ function $f_{r}$ is given by

$$
f_{r}(x)=\tan ^{r} x, \quad x \in I .
$$

Proof. We first show that $[0, \pi / 2) \subseteq I$ and for any $r \geq 1$ the following holds

$$
f_{r}(x) \leq \tan ^{r} x, \quad \forall x \in(0, \pi / 2) .
$$

For any $x \in I$ we have $f_{1}^{\prime}(x)=f_{0}(x)+f_{2}(x) \leq 1+f_{1}(x)^{2}$. Lemma 4.4 gives us that $\lim _{x \rightarrow 0} f_{1}(x)=0$ and then by integrating the last inequality we obtain that $\arctan f_{1}(x) \leq x$. Thus $f_{1}(x) \leq \tan x$. For $r \geq 2$ similar estimates hold since $f_{r}(x) \leq\left(f_{1}(x)\right)^{r}$. 
Let $S=\{(x, y) \in(0, \pi / 2) \times \mathbb{R}|| y \tan x \mid<1\}$. Using the properties of functions $f_{r}$ above we get that function $g$ defined by

$$
g(x, y)=f_{1}(x)+f_{2}(x) y+f_{3}(x) y^{2}+\cdots
$$

is well defined and differentiable on $S$. Explicit computations show that $g$ satisfies the following first order equation

$$
\left(y^{2}+1\right) g_{y}(x, y)-g_{x}(x, y)=-2 y g(x, y)-1, \quad \forall(x, y) \in S .
$$

In order to solve it we need some boundary conditions. Observe that since $f_{r}(x) \leq \tan ^{r} x$ for all $r \geq 1$, function $g$ satisfies $\lim _{x \rightarrow 0} g(x, y)=0$ for all $y \in \mathbb{R}$. Solving the above equation by the method of characteristics we obtain that

$$
g(x, y)=\frac{\tan x}{1-y \tan x} .
$$

Developing in $y$ power series we get that $f_{r}(x)=\tan ^{r} x$, as claimed. In particular, $f_{1}(x)=\tan x$. Since $f_{1}$ is increasing we also obtain that $I=(0, \pi / 2)$ and the proof is finished.

\subsection{Proofs of the two lemmas}

We are now able to prove Lemma 2.3 and Lemma 2.4.

Proof of Lemma 2.3. By definition of $E$ and Theorem 4.1 we have that

$$
\begin{aligned}
\left\|Q_{n+1}\right\|_{A P} & =\left\|E\left(\left|d_{1}\right|, \ldots,\left|d_{n}\right|\right)\right\|=\left\|R\left(\operatorname{arctanh}\left|d_{1}\right|, \ldots, \operatorname{arctanh}\left|d_{n}\right|\right)\right\| \\
& \leq f_{1}\left(\sum_{k=1}^{n} \operatorname{arctanh}\left|d_{k}\right|\right)=\tan \left(\sum_{k=1}^{n} \operatorname{arctanh}\left|d_{k}\right|\right) .
\end{aligned}
$$

Then estimate (2.17) follows.

Proof of Lemma 2.4. Let $\alpha>0$. By Theorem 4.1, $f_{1}(\alpha)=\tan \alpha$ if $\alpha<\pi / 2$ and $f_{1}(\alpha)=\infty$ if $\alpha \geq \pi / 2$. This means that for any $\varepsilon, N>0$ there exists a fine enough partition $t=\left(t_{1}, \ldots, t_{n-1}\right) \in A_{\alpha}$ such that $\|R(t)\| \geq \tan \alpha-\varepsilon$ if $\alpha<\pi / 2$ and $\|R(t)\| \geq N$ if $\alpha \geq \pi / 2$. Choosing, if necessary, finer partitions we can assume that $t_{k}<\operatorname{arctanh} d$, $k=1, \ldots, n-1$.

Choosing $\tilde{d}_{k}=\tanh t_{k}, k=1, \ldots, n-1$, we have $0<\tilde{d}_{k} \leq d, \sum_{k=1}^{n-1} \operatorname{arctanh} \tilde{d}_{k}=\sum_{k=1}^{n-1} t_{k}=\alpha$ and

$$
\left\|E\left(\tilde{d}_{1}, \ldots, \tilde{d}_{n-1}\right)\right\|=\|R(t)\| \geq \tan \alpha-\varepsilon \quad \text { or } \quad N,
$$

corresponding to $\alpha<\pi / 2$ or $\alpha \geq \pi / 2$, respectively.

Then for any $\left\{c_{k}\right\}_{k=1}^{n-1}$ linearly independent over $\mathbb{Q}$ and any $\left\{d_{k}\right\}_{k=1}^{n-1}$ with $\left|d_{k}\right|=\tilde{d}_{k}$ we have by Lemma 4.1 that $\left\{Q_{k}\right\}_{k=1}^{n}$ defined in (2.16) satisfies

$$
\left\|Q_{n}\right\|_{A P}=\left\|E\left(\left|d_{1}\right|, \ldots,\left|d_{n-1}\right|\right)\right\|=\left\|E\left(\tilde{d}_{1}, \ldots, \tilde{d}_{n-1}\right)\right\| \geq \tan \alpha-\varepsilon \quad \text { or } \quad N,
$$

accordingly, and the proof is finished.

\section{Conflict of interest statement}

Authors declare that there is no conflict of interest.

\section{Acknowledgements}

L.I. was partially supported by Grant PN-II-ID-PCE-2011-3-0075 of the Romanian National Authority for Scientific Research, CNCS - UEFISCDI (grant PN-II-ID-PCE-2011-3-0075), by Grants MTM2011-29306-C02-00 and MTM2014-52347 of MICINN, Spain, ERC Advanced Grant FP7-246775 NUMERIWAVES and FA9550-15-1-0027 of AFOSR. 
E.Z. was supported by the Advanced Grant FP7-246775 NUMERIWAVES of the European Research Council Executive Agency, FA9550-14-1-0214 of the EOARD-AFOSR, FA9550-15-1-0027 of AFOSR, the BERC 2014-2017 program of the Basque Government, the MTM2011-29306-C02-00, MTM2014-52347 and SEV-2013-0323 Grants of the MINECO and a Humboldt Award at the University of Erlangen-Nürnberg.

\section{References}

[1] V. Banica, Dispersion and Strichartz inequalities for Schrödinger equations with singular coefficients, SIAM J. Math. Anal. 35 (4) (2003) 868-883.

[2] N. Burq, F. Planchon, Smoothing and dispersive estimates for 1D Schrödinger equations with BV coefficients and applications, J. Funct. Anal. 236 (1) (2006) 265-298.

[3] C. Corduneanu, Almost Periodic Oscillations and Waves, Springer, New York, 2009.

[4] L. Demanet, G. Peyré, Compressive wave computation, Found. Comput. Math. 11 (3) (2011) 257-303.

[5] J.-P. Fouque, J. Garnier, G. Papanicolaou, K. Sølna, Wave Propagation and Time Reversal in Randomly Layered Media, Stochastic Modelling and Applied Probability, vol. 56, Springer, New York, 2007.

[6] L. Grafakos, Classical Fourier Analysis, second edition, Graduate Texts in Mathematics, vol. 249, Springer, New York, 2008. 